\title{
A GENERAL PERTURBATION FORMULA FOR ELECTROMAGNETIC FIELDS IN PRESENCE OF LOW VOLUME SCATTERERS
}

\author{
Roland GRIESMaier ${ }^{1}$
}

\begin{abstract}
In several practically interesting applications of electromagnetic scattering theory like, e.g., scattering from small point-like objects such as buried artifacts or small inclusions in non-destructive testing, scattering from thin curve-like objects such as wires or tubes, or scattering from thin sheet-like objects such as cracks, the volume of the scatterers is small relative to the volume of the surrounding medium and with respect to the wave length of the applied electromagnetic fields. This smallness typically causes problems when solving direct scattering problems due to the need to discretize the objects and also when solving inverse scattering problems because small objects have very little effect on electromagnetic fields. In this paper we consider an asymptotic representation formula for scattered electromagnetic waves caused by low volume objects contained in some otherwise homogeneous threedimensional bounded domain, assuming only that the scatterers are measurable and well-separated from the boundary of the domain. The formula yields a very general asymptotic model for electromagnetic scattering due to low volume objects that can either be used to simulate the corresponding electromagnetic fields or as the foundation of efficient reconstruction methods for inverse scattering problems with low volume scatterers. Our analysis extends results originally obtained in [Y. Capdeboscq and M.S. Vogelius, A general representation formula for boundary voltage perturbations caused by internal conductivity inhomogeneities of low volume fraction. Math. Model. Numer. Anal. 37 (2003) 159-173] for steady state voltage potentials to time-harmonic Maxwell's equations.
\end{abstract}

Mathematics Subject Classification. 35C20, 35Q60, 35J20.

Received August 31, 2010.

Published online July 22, 2011.

\section{INTRODUCTION}

In this work we study time-harmonic electromagnetic waves in a smoothly bounded domain filled with a homogeneous medium, which we call the background domain and accordingly the background medium. Supposing that this domain contains a penetrable object, the scatterer, that is a subdomain on which the electromagnetic properties of the medium differ from that of the background medium, we want to describe the influence of this object on electromagnetic fields under the additional assumption that its volume is small. Our main motivation to do so stems from inverse scattering problems, where one aims to recover the position and the shape of the scatterer from measurements of electromagnetic waves that are scattered by this object. If the volume of the object is small, it is well known that it has very little effect on electromagnetic fields, i.e., on the measurement

Keywords and phrases. Perturbation formulas, electromagnetic scattering, low volume scatterers, asymptotic expansions.

1 Department of Mathematical Sciences, University of Delaware, Newark, Delaware 19716, USA. griesmai@math.udel.edu 
data given in the inverse scattering problem. Therefore it is advisable to include all available a priori information on the structure of perturbations of electromagnetic fields due to low volume scatterers, when designing methods to reconstruct such objects from scattering data.

The aim of this article is to provide such a priori information for a very general class of low volume scatterers. For this purpose we consider the asymptotic behavior of scattered electromagnetic fields, i.e., the difference between the fields with and without scatterer, as the volume of the scatterer tends to zero. Our only regularity assumptions on the scatterer are that it is measurable and well separated from the boundary of the background domain. Observing that away from the scatterer the scattered field decreases at the same rate as the volume of the scatterer, we derive an analytic expression for the leading order term in the corresponding asymptotic expansion. Due to the generality of this formula higher order terms or even their precise order in terms of the volume of the scatterer are not obtained. For time-harmonic Maxwell's equations such an expansion has so far only been studied for the special case of diametrically small scatterers by Ammari, Vogelius, and Volkov [9] (see also [28] for a corresponding result for perfectly conducting scatterers in an unbounded layered background medium and Ammari and Kang [4] or Ammari and Khelifi [6] for higher order expansions in a two-dimensional setting).

Our analysis extends the work of Capdeboscq and Vogelius [18], where a similar representation formula has been established for perturbations in electrostatic potentials due to low volume conductivity inhomogeneities (cf. [19-21] for further studies in this direction). This formula generalizes and unifies earlier results obtained for the special case of diametrically small conductivity inhomogeneities (see, e.g., Friedman and Vogelius [26], Cedio-Fengya, Moskow, and Vogelius [22], or Ammari and Kang [3]) as well as for conductivity inhomogeneities of small thickness (see, e.g., Beretta et al. $[15,16]$ ). Similarly, the general formula for time-harmonic Maxwell's equations discussed in this article allows to recover the formula for diametrically small scatterers from [9] but also yields new asymptotic formulas for practically important cases like thin tubes or thin penetrable cracks mentioned before. Furthermore, our results can be extended to unbounded domains straightforwardly as done for diametrically small scatterers by Ammari and Volkov in [11].

The asymptotic formulas for diametrically small conductivity inhomogeneities and scatterers known so far form the foundation of several efficient reconstruction methods for inverse conductivity problems (see, e.g., Ammari, Moskow, and Vogelius [7], Ammari and Seo [8] or Brühl, Hanke, and Vogelius [17]) and inverse scattering problems for Maxwell's equations (see, e.g., Ammari et al. [2], Iakovleva et al. [33], Volkov [42], or $[28,29,31,32]$ ). In addition the general formula for electrostatic potentials from [18] has recently been used to investigate inverse conductivity problems for wires and tubes (see Beretta et al. [13] or [30]). Similarly, the general formula for electromagnetic fields considered here gives a new approach to develop efficient reconstruction methods for practically interesting inverse scattering problems, such as, e.g., inverse scattering for penetrable cracks (cf., e.g., Beretta and Francini [14] and Park and Lesselier [39] for an earlier study in this direction in a two-dimensional setting) or inverse scattering for thin tubular scatterers.

The arguments we use to establish the asymptotic representation formula rest on those applied in [18], suitably modified for the non-coercive Maxwell's equations and vector-valued functions. Among others the proof involves a representation formula for the scattered electromagnetic field in terms of Green's functions, energy estimates for electromagnetic fields obtained by duality arguments, so-called corrector potentials and polarization tensors, and an integration by parts technique that goes back to Murat and Tartar [38]. Applying two types of Helmholtz decompositions to the electromagnetic fields the gradient parts can be treated similarly to [18], while the main contribution of this work is the analysis of the divergence free parts that requires different techniques and is slightly more involved. In case of homogeneous background media, the polarization tensors turn out to be equivalent to the polarization tensor appearing in the electrostatic case, and thus earlier results on the properties of these tensors (see, e.g., $[5,18,19,21]$ ) immediately carry over to our setting. For historical remarks on polarization tensors and alternative approaches to establish asymptotic expansions of similar type as considered here we refer to the monographs by Ammari and Kang [5] and Il'in [34].

For several particular geometries of the scatterer explicit characterizations of the polarization tensor are known. Then the asymptotic representation formula yields a fast way to approximate the scattered field due 
to such low volume objects without meshing the scatterer itself. This is, e.g., the case for small ellipsoidal scatterers (see [5]), thin cylindrical scatterers (see [13]), or thin sheet-like scatterers (see $[15,16]$ ).

This article is organized as follows. In the next section we specify our mathematical setting and state the main result of this work, the asymptotic perturbation formula for the electromagnetic field. In Section 3, we collect some estimates for the scattered magnetic field, estimates for corresponding asymptotic corrector potentials, and the definitions of the electric and magnetic polarization tensors that will be used in the proof of the asymptotic formula. This proof is carried out in Section 4. In Section 5 we discuss three particular examples and comment on how the corresponding formulas can be used to solve inverse scattering problems. Finally, in Section 6 we outline possible generalizations of our findings.

\section{The mathematical Setting}

Suppose $\Omega \subset \mathbb{R}^{3}$ is a bounded domain with smooth boundary $\partial \Omega$ and unit outward normal $\boldsymbol{\nu}$. We consider a homogeneous background medium with constant electric conductivity $\sigma_{0} \geq 0$, constant electric permittivity $\varepsilon_{0}>0$, and constant magnetic permeability $\mu_{0}>0$. A time-harmonic magnetic background field in this medium at a frequency $\omega / 2 \pi>0$ corresponding to boundary data

$$
\boldsymbol{g} \in \boldsymbol{H}_{\mathrm{div}}^{-1 / 2}(\partial \Omega):=\left\{\boldsymbol{f} \in H^{-1 / 2}\left(\partial \Omega, \mathbb{C}^{3}\right) \mid \operatorname{div}_{\partial \Omega} \boldsymbol{f} \in H^{-1 / 2}(\partial \Omega, \mathbb{C})\right\}
$$

is governed by the boundary value problem

$$
\begin{aligned}
\operatorname{curl}\left(\frac{1}{\tilde{\varepsilon}_{0}} \operatorname{curl} \boldsymbol{H}_{0}\right)-\omega^{2} \mu_{0} \boldsymbol{H}_{0} & =0 & & \text { in } \Omega, \\
\frac{1}{\tilde{\varepsilon}_{0}}\left(\operatorname{curl} \boldsymbol{H}_{0}\right) \times \boldsymbol{\nu} & =\boldsymbol{g} & & \text { on } \partial \Omega .
\end{aligned}
$$

Here and in the following we write $\tilde{\varepsilon}_{0}:=\varepsilon_{0}+\mathrm{i} \sigma_{0} / \omega$. Accordingly the electric background field is given by $\boldsymbol{E}_{0}=\left(\mathrm{i} / \omega \tilde{\varepsilon}_{0}\right) \operatorname{curl} \boldsymbol{H}_{0}$.

Next, let $\Omega_{0} \subset \subset \Omega$ be well separated from $\partial \Omega$, i.e., $\operatorname{dist}\left(\Omega_{0}, \partial \Omega\right) \geq d_{0}$ for some constant $d_{0}>0$, and denote by $\left(D_{\rho_{n}}\right)_{n \in \mathbb{N}}$ a family of measurable subsets of $\Omega$ satisfying $\lim _{n \rightarrow \infty}\left|D_{\rho_{n}}\right|=0$, where $\left|D_{\rho_{n}}\right|$ denotes the Lebesgue measure of $D_{\rho_{n}}$. Each $D_{\rho_{n}}$ is considered as a scatterer contained in $\Omega$ causing a discontinuous permeability and (complex-valued) permittivity

$$
\mu_{\rho_{n}}(x):=\left\{\begin{array}{ll}
\mu_{1}(x), & x \in D_{\rho_{n}}, \\
\mu_{0}, & x \in \Omega \backslash \overline{D_{\rho_{n}}},
\end{array} \quad \tilde{\varepsilon}_{\rho_{n}}(x):= \begin{cases}\tilde{\varepsilon}_{1}(x), & x \in D_{\rho_{n}}, \\
\tilde{\varepsilon}_{0}, & x \in \Omega \backslash \overline{D_{\rho_{n}}}\end{cases}\right.
$$

where $\mu_{1} \in C^{\infty}(\bar{\Omega}, \mathbb{R})$ satisfies $0<c_{\mu_{1}} \leq \mu_{1} \leq C_{\mu_{1}}<\infty$ and $\tilde{\varepsilon}_{1}:=\varepsilon_{1}+\mathrm{i}\left(\sigma_{1} / \omega\right)$ with $\varepsilon_{1} \in C^{\infty}(\bar{\Omega}, \mathbb{R})$ and $\sigma_{1} \in C^{\infty}(\bar{\Omega}, \mathbb{R})$ such that $0<c_{\varepsilon_{1}} \leq \varepsilon_{1} \leq C_{\varepsilon_{1}}<\infty$ and $0 \leq \sigma_{1} \leq C_{\sigma_{1}}<\infty$ for some constants $c_{\mu_{1}}, C_{\mu_{1}}, c_{\varepsilon_{1}}, C_{\varepsilon_{1}}$, and $C_{\sigma_{1}}$. The magnetic field in presence of these scatterers corresponding to the same boundary data as above satisfies

$$
\begin{aligned}
\operatorname{curl}\left(\frac{1}{\tilde{\varepsilon}_{\rho_{n}}} \operatorname{curl} \boldsymbol{H}_{\rho_{n}}\right)-\omega^{2} \mu_{\rho_{n}} \boldsymbol{H}_{\rho_{n}} & =0 & \text { in } \Omega, \\
\frac{1}{\tilde{\varepsilon}_{\rho_{n}}}\left(\operatorname{curl} \boldsymbol{H}_{\rho_{n}}\right) \times \boldsymbol{\nu} & =\boldsymbol{g} & \text { on } \partial \Omega .
\end{aligned}
$$

Introducing the sesquilinear forms $a_{0}$ and $a_{\rho_{n}}, n \in \mathbb{N}$, on $\boldsymbol{H}(\operatorname{curl}, \Omega) \times \boldsymbol{H}(\operatorname{curl}, \Omega)$, where

$$
\boldsymbol{H}(\operatorname{curl}, \Omega):=\left\{\boldsymbol{u} \in \boldsymbol{L}^{2}\left(\Omega, \mathbb{C}^{3}\right) \mid \operatorname{curl} \boldsymbol{u} \in \boldsymbol{L}^{2}\left(\Omega, \mathbb{C}^{3}\right)\right\}
$$


by

$$
\begin{aligned}
a_{0}(\boldsymbol{u}, \boldsymbol{v}) & :=\int_{\Omega} \frac{1}{\tilde{\varepsilon}_{0}} \operatorname{curl} \boldsymbol{u} \cdot \overline{\operatorname{curl} \boldsymbol{v}} \mathrm{d} \boldsymbol{x}-\omega^{2} \int_{\Omega} \mu_{0} \boldsymbol{u} \cdot \overline{\boldsymbol{v}} \mathrm{d} \boldsymbol{x}, \\
a_{\rho_{n}}(\boldsymbol{u}, \boldsymbol{v}) & :=\int_{\Omega} \frac{1}{\tilde{\varepsilon}_{\rho_{n}}} \operatorname{curl} \boldsymbol{u} \cdot \overline{\operatorname{curl} \boldsymbol{v}} \mathrm{d} \boldsymbol{x}-\omega^{2} \int_{\Omega} \mu_{\rho_{n}} \boldsymbol{u} \cdot \overline{\boldsymbol{v}} \mathrm{d} \boldsymbol{x},
\end{aligned}
$$

the weak formulations of the boundary value problems (2.1) and (2.2) ask to find $\boldsymbol{H}_{0}, \boldsymbol{H}_{\rho_{n}} \in \boldsymbol{H}(\mathbf{c u r l}, \Omega)$ such that

$$
\begin{array}{rlr}
a_{0}\left(\boldsymbol{H}_{0}, \boldsymbol{v}\right) & =\int_{\partial \Omega} \boldsymbol{g} \cdot \overline{\boldsymbol{v}} \mathrm{d} s, & \text { for all } \boldsymbol{v} \in \boldsymbol{H}(\operatorname{curl}, \Omega), \\
a_{\rho_{n}}\left(\boldsymbol{H}_{\rho_{n}}, \boldsymbol{v}\right) & =\int_{\partial \Omega} \boldsymbol{g} \cdot \overline{\boldsymbol{v}} \mathrm{d} s, & \text { for all } \boldsymbol{v} \in \boldsymbol{H}(\operatorname{curl}, \Omega),
\end{array}
$$

respectively. Throughout we assume that (2.3) has a unique solution. In this case

$$
\left\|\boldsymbol{H}_{0}\right\|_{\boldsymbol{H}(\mathbf{c u r l}, \Omega)} \leq C\|\boldsymbol{g}\|_{\boldsymbol{H}_{\mathrm{div}}^{-1 / 2}(\partial \Omega)}
$$

and regularity results for Maxwell's equations ( $c f .$, e.g., Weber [43]) guarantee that $\boldsymbol{H}_{0}$ is smooth in $\Omega_{0}$. It has been shown in [9] for the special case of diametrically small scatterers that uniqueness of solutions to (2.3) implies existence and uniqueness of solutions to (2.4) provided that $\left|D_{\rho_{n}}\right|$ is small enough, i.e., $n$ is large enough. The proof of this result in [9] carries over to our setting straightforwardly.

Proposition 2.1. Assume that (2.3) has a unique solution. Then, there exists an upper bound $R>0$ such that for any $\boldsymbol{g} \in \boldsymbol{H}_{\mathrm{div}}^{-1 / 2}(\partial \Omega)$ and any $n \in \mathbb{N}$ with $\left|D_{\rho_{n}}\right| \leq R$ the variational problem (2.4) has a unique solution $\boldsymbol{H}_{\rho_{n}} \in \boldsymbol{H}($ curl, $\Omega)$ satisfying

where the constant $C$ is independent of $n$.

$$
\left\|\boldsymbol{H}_{\rho_{n}}\right\|_{\boldsymbol{H}(\mathbf{c u r l}, \Omega)} \leq C\|\boldsymbol{g}\|_{\boldsymbol{H}_{\mathrm{div}}^{-1 / 2}(\partial \Omega)}
$$

We are interested in the asymptotic behavior of $\boldsymbol{\nu} \times\left.\left(\boldsymbol{H}_{\rho_{n}}-\boldsymbol{H}_{0}\right)\right|_{\partial \Omega}$ as $\left|D_{\rho_{n}}\right| \rightarrow 0$. Following [18] we start by observing that for all $n \in \mathbb{N}$ the positive regular Borel measure

$$
\mu_{n}(E):=\int_{E}\left|D_{\rho_{n}}\right|^{-1} \chi_{\rho_{n}} \mathrm{~d} \boldsymbol{x}, \quad E \subset \Omega \text { Borel measurable, }
$$

satisfies $\left|\mu_{n}\right| \leq 1$, where $\left|\mu_{n}\right|$ denotes the total variation of $\mu_{n}$ and $\chi_{\rho_{n}}$ is the characteristic function of $D_{\rho_{n}}$. This means that the sequence $\left(\mu_{n}\right)_{n \in \mathbb{N}}$ is bounded in the space $M(\Omega, \mathbb{C})$ of complex regular Borel measures on $\Omega$. By Riesz's representation theorem $M(\Omega, \mathbb{C})$ is isomorphic to the dual space of $C_{0}(\Omega, \mathbb{C})$ of continuous functions on $\Omega$ that vanish on $\partial \Omega$ (cf., e.g., Rudin [40], Thm. 6.19). Thus the Banach-Alaoglu Theorem (cf., e.g., Rudin [41], Thm. 3.15) guarantees the existence of a subsequence, also denoted by $\left(D_{\rho_{n}}\right)_{n \in \mathbb{N}}$, and a complex regular Borel measure $\mu$ such that for every $\phi \in C_{0}(\Omega, \mathbb{C})$,

$$
\lim _{n \rightarrow \infty} \int_{\Omega} \phi \mathrm{d} \mu_{n}=\int_{\Omega} \phi \mathrm{d} \mu .
$$

Another ingredient of the asymptotic perturbation formula established in Theorem 2.2 below, is the dyadic Green's function for time-harmonic Maxwell's equations corresponding to the homogeneous background medium,

$$
\mathbb{G}(\boldsymbol{x}, \boldsymbol{y}):=\Phi_{k}(\boldsymbol{x}-\boldsymbol{y}) \mathbb{I}_{3}+\frac{1}{k^{2}} \nabla_{x} \operatorname{div}_{x}\left(\Phi_{k}(\boldsymbol{x}-\boldsymbol{y}) \mathbb{I}_{3}\right), \quad \boldsymbol{x}, \boldsymbol{y} \in \mathbb{R}^{3}, \boldsymbol{x} \neq \boldsymbol{y},
$$


where $\Phi_{k}(\boldsymbol{x}-\boldsymbol{y}):=e^{\mathrm{i} k|\boldsymbol{x}-\boldsymbol{y}|} / 4 \pi|\boldsymbol{x}-\boldsymbol{y}|$ is the fundamental solution of the Helmholtz equation with wave number $k:=\sqrt{\omega^{2} \tilde{\varepsilon}_{0} \mu_{0}}$ (if $\tilde{\varepsilon}_{0} \notin \mathbb{R}$, then $k$ is taken to have positive imaginary part) and $\mathbb{I}_{3}$ denotes the $3 \times 3$ identity matrix. Here and throughout this work we let scalar operators operate on vectors component-wise and vector operators on matrices column by column. Note that for any $\boldsymbol{y} \in \mathbb{R}^{3}$ the dyadic Green's function is the distributional solution of

$$
\operatorname{curl}_{x} \operatorname{curl}_{x} \mathbb{G}(\boldsymbol{x}, \boldsymbol{y})-k^{2} \mathbb{G}(\boldsymbol{x}, \boldsymbol{y})=\delta_{\boldsymbol{y}}(\boldsymbol{x}), \quad \boldsymbol{x} \in \mathbb{R}^{3}
$$

In the following theorem $\boldsymbol{L}^{2}\left(\Omega, \mathbb{K}^{3 \times 3} ; \mu\right), \mathbb{K}=\mathbb{R}$ or $\mathbb{K}=\mathbb{C}$, denotes the space of real or complex matrix-valued functions on $\Omega$ that are square integrable with respect to the regular Borel measure $\mu$, respectively.

Theorem 2.2. Suppose $\left(D_{\rho_{n}}\right)_{n \in \mathbb{N}}$ is a sequence of measurable subsets of $\Omega_{0} \subset \subset \Omega$ as introduced above and assume that $\left|D_{\rho_{n}}\right| \leq R$ for all $n \in \mathbb{N}$, where $R$ is the upper bound from Proposition 2.1. Given $\boldsymbol{g} \in \boldsymbol{H}_{\mathrm{div}}^{-1 / 2}(\partial \Omega)$ let $\boldsymbol{H}_{0}$ and $\boldsymbol{H}_{\rho_{n}}, n \in \mathbb{N}$, denote the corresponding solutions of (2.3) and (2.4), respectively. Then, there exists a subsequence, also denoted by $\left(D_{\rho_{n}}\right)_{n \in \mathbb{N}}$, a positive regular Borel measure $\mu$ and matrix-valued functions $\mathbb{M}^{\tilde{\varepsilon}} \in \boldsymbol{L}^{2}\left(\Omega, \mathbb{C}^{3 \times 3} ; \mu\right)$ and $\mathbb{M}^{\mu} \in \boldsymbol{L}^{2}\left(\Omega, \mathbb{R}^{3 \times 3} ; \mu\right)$, called electric and magnetic polarization tensors, respectively, such that for $\boldsymbol{y} \in \partial \Omega$,

$$
\begin{aligned}
\boldsymbol{\nu}(\boldsymbol{y}) \times\left(\boldsymbol{H}_{\rho_{n}}-\boldsymbol{H}_{0}\right)(\boldsymbol{y})-2 \int_{\partial \Omega}\left(\boldsymbol{\nu}(\boldsymbol{y}) \times \operatorname{curl}_{x} \mathbb{G}(\cdot, \boldsymbol{y})\right)\left(\boldsymbol{\nu} \times\left(\boldsymbol{H}_{\rho_{n}}-\boldsymbol{H}_{0}\right)\right) \mathrm{d} s \\
=\left|D_{\rho_{n}}\right| 2 \tilde{\varepsilon}_{0}\left(-\int_{\Omega} \frac{\tilde{\varepsilon}_{1}}{\tilde{\varepsilon}_{0}}\left(\frac{1}{\tilde{\varepsilon}_{0}}-\frac{1}{\tilde{\varepsilon}_{1}}\right)\left(\boldsymbol{\nu}(\boldsymbol{y}) \times \operatorname{curl}_{x} \mathbb{G}(\cdot, \boldsymbol{y})\right) \mathbb{M}^{\tilde{\varepsilon}} \operatorname{curl} \boldsymbol{H}_{0} \mathrm{~d} \mu\right. \\
\left.\quad+\omega^{2} \int_{\Omega}\left(\mu_{0}-\mu_{1}\right)(\boldsymbol{\nu}(\boldsymbol{y}) \times \mathbb{G}(\cdot, \boldsymbol{y})) \mathbb{M}^{\mu} \boldsymbol{H}_{0} \mathrm{~d} \mu\right)+o\left(\left|D_{\rho_{n}}\right|\right) .
\end{aligned}
$$

The subsequence $\left(D_{\rho_{n}}\right)_{n \in \mathbb{N}}$ and the functions $\mathbb{M}^{\tilde{\varepsilon}}$ and $\mathbb{M}^{\mu}$ are independent of $\boldsymbol{g}$. The last term on the right hand side of (2.7) satisfies

$$
\lim _{n \rightarrow \infty}\left\|o\left(\left|D_{\rho_{n}}\right|\right)\right\|_{L^{\infty}\left(\partial \Omega, \mathbb{C}^{3}\right)} /\left|D_{\rho_{n}}\right|=0
$$

for any $\boldsymbol{g} \in \boldsymbol{H}_{\mathrm{div}}^{-1 / 2}(\partial \Omega)$, uniformly on bounded subsets of $\boldsymbol{H}_{\mathrm{div}}^{-1 / 2}(\partial \Omega)$.

Although in this work we consider the magnetic field only, we note that an asymptotic perturbation formula similar to (2.7) can be established for the electric field as well.

Remark 2.3 (Polarization Tensors). Before we give a precise definition of the electric and magnetic polarization tensor in Section 3.3 below, we recall in the following two important properties of these matrix valued functions. It has been shown in [18], Section 4, that the magnetic polarization tensor $\mathbb{M}^{\mu}$ (and similarly the electric polarization tensor $\mathbb{M} \tilde{\varepsilon}^{\tilde{\varepsilon}}$, provided that $\tilde{\varepsilon}_{0}, \tilde{\varepsilon}_{1} \in \mathbb{R}$, i.e., $\sigma_{0}=\sigma_{1}=0$ ) is symmetric and positive definite in the sense that for $\mu$-a.e. $\boldsymbol{x} \in \Omega$,

$$
\begin{gathered}
\mathbb{M}^{\mu^{\top}}(\boldsymbol{x})=\mathbb{M}^{\mu}(\boldsymbol{x}), \\
\min \left\{1, \frac{\mu_{1}(\boldsymbol{x})}{\mu_{0}(\boldsymbol{x})}\right\}|\boldsymbol{\xi}|^{2} \leq \boldsymbol{\xi}^{\top} \mathbb{M}^{\mu}(\boldsymbol{x}) \boldsymbol{\xi} \leq \max \left\{1, \frac{\mu_{1}(\boldsymbol{x})}{\mu_{0}(\boldsymbol{x})}\right\}|\boldsymbol{\xi}|^{2}
\end{gathered}
$$

for all $\boldsymbol{\xi} \in \mathbb{R}^{3}$. For dissipative media, following the proof for real-valued coefficients in [18], Section 4, we obtain that for $\mu$-a.e. $\boldsymbol{x} \in \Omega$ the electric polarization tensor $\mathbb{M} \tilde{\varepsilon}(\boldsymbol{x})$ satisfies

$$
\mathbb{M}^{\tilde{\varepsilon}^{\top}}(\boldsymbol{x})=\mathbb{M}^{\tilde{\varepsilon}}(\boldsymbol{x}),
$$


i.e., it is symmetric but not Hermitian, and

$$
\begin{aligned}
& \left(\operatorname{Re}\left(\tilde{\varepsilon}_{1}-\tilde{\varepsilon}_{0}\right)-\left|\frac{\left(\tilde{\varepsilon}_{1}-\tilde{\varepsilon}_{0}\right)^{2}}{\tilde{\varepsilon}_{1}}\right|\right)|\boldsymbol{\xi}|^{2} \leq \boldsymbol{\xi}^{\top} \operatorname{Re}\left(\left(\tilde{\varepsilon}_{1}-\tilde{\varepsilon}_{0}\right) \mathbb{M}^{\tilde{\varepsilon}}(\boldsymbol{x})\right) \boldsymbol{\xi} \leq \operatorname{Re}\left(\tilde{\varepsilon}_{1}-\tilde{\varepsilon}_{0}\right)|\boldsymbol{\xi}|^{2} \\
& \left(\operatorname{Im}\left(\tilde{\varepsilon}_{1}-\tilde{\varepsilon}_{0}\right)-\left|\frac{\left(\tilde{\varepsilon}_{1}-\tilde{\varepsilon}_{0}\right)^{2}}{\tilde{\varepsilon}_{1}}\right|\right)|\boldsymbol{\xi}|^{2} \leq \boldsymbol{\xi}^{\top} \operatorname{Im}\left(\left(\tilde{\varepsilon}_{1}-\tilde{\varepsilon}_{0}\right) \mathbb{M}^{\tilde{\varepsilon}}(\boldsymbol{x})\right) \boldsymbol{\xi} \leq \operatorname{Im}\left(\tilde{\varepsilon}_{1}-\tilde{\varepsilon}_{0}\right)|\boldsymbol{\xi}|^{2}
\end{aligned}
$$

for all $\boldsymbol{\xi} \in \mathbb{R}^{3}$, i.e., it is uniformly bounded.

\section{Preliminary convergence estimates}

In this section we derive energy estimates for the difference $\boldsymbol{H}_{\rho_{n}}-\boldsymbol{H}_{0}, n \in \mathbb{N}$, of the magnetic fields with and without scatterers and for the difference of corresponding asymptotic corrector potentials introduced below. Moreover, we define the electric and magnetic polarization tensors used in this work and discuss their relation to the polarization tensor appearing in the asymptotic representation formula for electrostatic potentials from $[18,19,21]$. Throughout we assume that $\left(D_{\rho_{n}}\right)_{n \in \mathbb{N}}$ is a sequence of measurable subsets of $\Omega_{0} \subset \subset \Omega$ as in Section 2 such that $\left|D_{\rho_{n}}\right| \leq R$, where $R$ is the upper bound from Proposition 2.1, and that (2.6) is satisfied.

\subsection{Two decompositions of $\boldsymbol{H}(\operatorname{curl}, \Omega)$}

The energy estimates will be formulated in terms of two types of Helmholtz decompositions of $\boldsymbol{H}(\mathbf{c u r l}, \Omega)$ related to the variational formulations (2.3) and (2.4), respectively. Following [9], we define the spaces $\boldsymbol{Y}:=$ $\nabla H^{1}(\Omega, \mathbb{C})$,

$$
\boldsymbol{Y}_{0}^{\perp}:=\left\{\boldsymbol{u} \in \boldsymbol{H}(\operatorname{curl}, \Omega) \mid \operatorname{div}\left(\mu_{0} \boldsymbol{u}\right)=0 \text { in } \Omega, \boldsymbol{\nu} \cdot \boldsymbol{u}=0 \text { on } \partial \Omega\right\}
$$

and for all $n \in \mathbb{N}$,

$$
\boldsymbol{Y}_{\rho_{n}}^{\perp}:=\left\{\boldsymbol{u} \in \boldsymbol{H}(\operatorname{curl}, \Omega) \mid \operatorname{div}\left(\mu_{\rho_{n}} \boldsymbol{u}\right)=0 \text { in } \Omega, \boldsymbol{\nu} \cdot \boldsymbol{u}=0 \text { on } \partial \Omega\right\} .
$$

It is well known that the subspace $\boldsymbol{Y}$ is closed in $\boldsymbol{H}(\operatorname{curl}, \Omega)$. Furthermore, $\boldsymbol{Y}_{0}^{\perp}$ and $\boldsymbol{Y}_{\rho_{n}}^{\perp}$ are the orthogonal complements of $\boldsymbol{Y}$ in $\boldsymbol{H}(\mathbf{c u r l}, \Omega)$ with respect to the inner products

$$
\langle\boldsymbol{u}, \boldsymbol{v}\rangle_{0}:=\int_{\Omega} \mu_{0} \boldsymbol{u} \cdot \overline{\boldsymbol{v}} \mathrm{d} \boldsymbol{x} \quad \text { and } \quad\langle\boldsymbol{u}, \boldsymbol{v}\rangle_{\rho_{n}}:=\int_{\Omega} \mu_{\rho_{n}} \boldsymbol{u} \cdot \overline{\boldsymbol{v}} \mathrm{d} \boldsymbol{x},
$$

$\boldsymbol{u}, \boldsymbol{v} \in \boldsymbol{H}(\mathbf{c u r l}, \Omega)$, respectively. This yields decompositions

$$
\boldsymbol{H}(\operatorname{curl}, \Omega)=\boldsymbol{Y} \oplus \boldsymbol{Y}_{0}^{\perp}=\boldsymbol{Y} \oplus \boldsymbol{Y}_{\rho_{n}}^{\perp}, \quad n \in \mathbb{N},
$$

and corresponding orthogonal projections

$$
P_{0}: \boldsymbol{H}(\operatorname{curl}, \Omega) \rightarrow \boldsymbol{Y}_{0}^{\perp} \quad \text { and } \quad P_{\rho_{n}}: \boldsymbol{H}(\operatorname{curl}, \Omega) \rightarrow \boldsymbol{Y}_{\rho_{n}}^{\perp},
$$

given by $P_{0} \boldsymbol{u}:=\boldsymbol{u}-\nabla p_{0}$ and $P_{\rho_{n}} \boldsymbol{u}=\boldsymbol{u}-\nabla p_{\rho_{n}}$, where $p_{0}, p_{\rho_{n}} \in H_{\diamond}^{1}(\Omega, \mathbb{C}):=\left\{\phi \in H^{1}(\Omega, \mathbb{C}) \mid \int_{\partial \Omega} \phi \mathrm{d} s=0\right\}$ satisfy

$$
\begin{aligned}
\int_{\Omega} \mu_{0} \nabla p_{0} \cdot \overline{\nabla \phi} \mathrm{d} \boldsymbol{x}=\int_{\Omega} \mu_{0} \boldsymbol{u} \cdot \overline{\nabla \phi} \mathrm{d} \boldsymbol{x} & \text { for all } \phi \in H^{1}(\Omega, \mathbb{C}), \\
\int_{\Omega} \mu_{\rho_{n}} \nabla p_{\rho_{n}} \cdot \overline{\nabla \phi} \mathrm{d} \boldsymbol{x}=\int_{\Omega} \mu_{\rho_{n}} \boldsymbol{u} \cdot \overline{\nabla \phi} \mathrm{d} \boldsymbol{x} & \text { for all } \phi \in H^{1}(\Omega, \mathbb{C})
\end{aligned}
$$


respectively. For $\boldsymbol{v} \in \boldsymbol{Y}_{0}^{\perp}$ the Friedrichs inequality

$$
\|\boldsymbol{u}\|_{\boldsymbol{L}^{2}\left(\Omega, \mathbb{C}^{3}\right)} \leq C\|\operatorname{curl} \boldsymbol{u}\|_{\boldsymbol{L}^{2}\left(\Omega, \mathbb{C}^{3}\right)}
$$

follows directly from [37], Corollary 3.51 in the book of Monk and using the compactness result [9], Proposition 3 it can be shown that (3.3) holds for $\boldsymbol{u} \in \boldsymbol{Y}_{\rho_{n}}^{\perp}$ as well.

Accordingly we can decompose

$$
\boldsymbol{H}_{0}=: \boldsymbol{h}_{0}+\nabla q_{0} \quad \text { and } \quad \boldsymbol{H}_{\rho_{n}}=: \boldsymbol{h}_{\rho_{n}}+\nabla q_{\rho_{n}}, \quad n \in \mathbb{N}
$$

such that $\boldsymbol{h}_{0} \in \boldsymbol{Y}_{0}^{\perp}, \boldsymbol{h}_{\rho_{n}} \in \boldsymbol{Y}_{\rho_{n}}^{\perp}$, and $q_{0}, q_{\rho_{n}} \in H_{\diamond}^{1}(\Omega, \mathbb{C})$. Combining the weak formulations $(2.3)$, (2.4), and (3.2) it follows immediately that $q_{0}$ and $q_{\rho_{n}}$ are weak solutions of

$$
\begin{aligned}
\operatorname{div}\left(\mu_{0} \nabla q_{0}\right) & =0 & & \text { in } \Omega, \\
\mu_{0} \frac{\partial q_{0}}{\partial \boldsymbol{\nu}} & =\frac{1}{\omega^{2}} \operatorname{div}_{\partial \Omega} \boldsymbol{g} & & \text { on } \partial \Omega,
\end{aligned}
$$

and

$$
\begin{aligned}
\operatorname{div}\left(\mu_{\rho_{n}} \nabla q_{\rho_{n}}\right) & =0 & & \text { in } \Omega \\
\mu_{\rho_{n}} \frac{\partial q_{\rho_{n}}}{\partial \boldsymbol{\nu}} & =\frac{1}{\omega^{2}} \operatorname{div}_{\partial \Omega} \boldsymbol{g} & & \text { on } \partial \Omega
\end{aligned}
$$

respectively. Thus, regularity results for elliptic equations show that the solution $q_{0}$ is smooth in $\Omega_{0}$ (cf. McLean [36], Thm. 4.18).

Sometimes we will also use the slightly different decompositions

$$
\boldsymbol{H}_{0}=: \boldsymbol{h}_{0}^{\left(\rho_{n}\right)}+\nabla q_{0}^{\left(\rho_{n}\right)} \quad \text { and } \quad \boldsymbol{H}_{\rho_{n}}=: \boldsymbol{h}_{\rho_{n}}^{(0)}+\nabla q_{\rho_{n}}^{(0)}, \quad n \in \mathbb{N}
$$

with $\boldsymbol{h}_{0}^{\left(\rho_{n}\right)} \in \boldsymbol{Y}_{\rho_{n}}^{\perp}, \boldsymbol{h}_{\rho_{n}}^{(0)} \in \boldsymbol{Y}_{0}^{\perp}$, and $q_{0}^{\left(\rho_{n}\right)}, q_{\rho_{n}}^{(0)} \in H_{\diamond}^{1}(\Omega, \mathbb{C})$. Note that $q_{0}^{\left(\rho_{n}\right)}$ and $q_{\rho_{n}}^{(0)}$ are weak solutions of

$$
\begin{aligned}
\operatorname{div}\left(\mu_{\rho_{n}} \nabla q_{0}^{\left(\rho_{n}\right)}\right) & =\operatorname{div}\left(\mu_{\rho_{n}} \boldsymbol{H}_{0}\right) & & \text { in } \Omega \\
\mu_{\rho_{n}} \frac{\partial q_{0}^{\left(\rho_{n}\right)}}{\partial \boldsymbol{\nu}} & =\frac{1}{\omega^{2}} \operatorname{div}_{\partial \Omega} \boldsymbol{g} & & \text { on } \partial \Omega
\end{aligned}
$$

and

$$
\begin{aligned}
\operatorname{div}\left(\mu_{0} \nabla q_{\rho_{n}}^{(0)}\right) & =\operatorname{div}\left(\mu_{0} \boldsymbol{H}_{\rho_{n}}\right) & & \text { in } \Omega \\
\mu_{0} \frac{\partial q_{\rho_{n}}^{(0)}}{\partial \boldsymbol{\nu}} & =\frac{1}{\omega^{2}} \operatorname{div}_{\partial \Omega} \boldsymbol{g} & & \text { on } \partial \Omega
\end{aligned}
$$

respectively.

\subsection{Estimates for the magnetic field}

To estimate the difference $\boldsymbol{H}_{\rho_{n}}-\boldsymbol{H}_{0}, n \in \mathbb{N}$, we consider its gradient part and its divergence free part according to the decompositions (3.4) and (3.7) separately. As already mentioned in the introduction, the analysis for the gradient part in this and the following sections follows closely the corresponding analysis for the electrostatic case from [18], while the divergence free parts require different arguments and techniques. Throughout we use generic constants $C$ and $C_{\eta}, \eta \in[1 / 5,1 / 2]$, the values of which might change from line to line. 
Lemma 3.1. Let $q_{0}$ and $q_{\rho_{n}}$ be as in (3.4). Then, there exists a constant $C$ such that for any $n \in \mathbb{N}$,

$$
\left\|q_{\rho_{n}}-q_{0}\right\|_{H^{1}(\Omega, \mathbb{C})} \leq C\left|D_{\rho_{n}}\right|^{1 / 2}\|\boldsymbol{g}\|_{\boldsymbol{H}_{\mathrm{div}}^{-1 / 2}(\partial \Omega)},
$$

and for any $\eta \in[1 / 5,1 / 2]$ there exists a constant $C_{\eta}$ such that

$$
\left\|q_{\rho_{n}}-q_{0}\right\|_{L^{2}(\Omega, \mathbb{C})} \leq C_{\eta}\left|D_{\rho_{n}}\right|^{1-\eta}\|\boldsymbol{g}\|_{\boldsymbol{H}_{\mathrm{div}}^{-1 / 2}(\partial \Omega)} .
$$

Proof. Since $q_{0}$ and $q_{\rho_{n}}$ satisfy (3.5) and (3.6), respectively, this lemma is a special case of [18], Lemma 1.

Lemma 3.2. Let $\boldsymbol{h}_{0}, \boldsymbol{h}_{0}^{\left(\rho_{n}\right)}, \boldsymbol{h}_{\rho_{n}}$, and $\boldsymbol{h}_{\rho_{n}}^{(0)}$ be as in (3.4) and (3.7), respectively. Then, there exists a constant $C$ such that for any $n \in \mathbb{N}$,

$$
\begin{aligned}
&\left\|\boldsymbol{h}_{\rho_{n}}-\boldsymbol{h}_{0}^{\left(\rho_{n}\right)}\right\|_{\boldsymbol{H}(\mathbf{c u r l}, \Omega)} \leq C\left|D_{\rho_{n}}\right|^{1 / 2}\|\boldsymbol{g}\|_{\boldsymbol{H}_{\mathrm{div}}^{-1 / 2}(\partial \Omega)}, \\
&\left\|\boldsymbol{h}_{0}-\boldsymbol{h}_{0}^{\left(\rho_{n}\right)}\right\|_{\boldsymbol{H}(\mathbf{c u r l}, \Omega)} \leq C\left|D_{\rho_{n}}\right|^{1 / 2}\|\boldsymbol{g}\|_{\boldsymbol{H}_{\mathrm{div}}^{-1 / 2}(\partial \Omega)} \\
&\left\|\boldsymbol{h}_{\rho_{n}}-\boldsymbol{h}_{\rho_{n}}^{(0)}\right\|_{\boldsymbol{H}(\mathbf{c u r l}, \Omega)} \leq C\left|D_{\rho_{n}}\right|^{1 / 2}\|\boldsymbol{g}\|_{\boldsymbol{H}_{\mathrm{div}}^{-1 / 2}(\partial \Omega)}
\end{aligned}
$$

Furthermore, for any $\eta \in[1 / 5,1 / 2]$ there exists a constant $C_{\eta}$ such that

$$
\left\|\boldsymbol{h}_{\rho_{n}}^{(0)}-\boldsymbol{h}_{0}\right\|_{\boldsymbol{L}^{2}\left(\Omega, \mathbb{C}^{3}\right)} \leq C_{\eta}\left|D_{\rho_{n}}\right|^{1-\eta}\|\boldsymbol{g}\|_{\boldsymbol{H}_{\mathrm{div}}^{-1 / 2}(\partial \Omega)} .
$$

Proof. Step 1 (proof of (3.12a)). From the weak formulations (2.3) and (2.4) we find that $\boldsymbol{H}_{0}=\boldsymbol{h}_{0}^{\left(\rho_{n}\right)}+\nabla q_{0}^{\left(\rho_{n}\right)}$ and $\boldsymbol{H}_{\rho_{n}}=\boldsymbol{h}_{\rho_{n}}+\nabla q_{\rho_{n}}$ satisfy

$$
\begin{aligned}
\int_{\Omega} \frac{1}{\tilde{\varepsilon}_{\rho_{n}}} \operatorname{curl}\left(\boldsymbol{H}_{\rho_{n}}-\boldsymbol{H}_{0}\right) \cdot \overline{\operatorname{curl} \boldsymbol{u}} \mathrm{d} \boldsymbol{x}-\omega^{2} \int_{\Omega} \mu_{\rho_{n}}\left(\boldsymbol{H}_{\rho_{n}}-\boldsymbol{H}_{0}\right) \cdot \overline{\boldsymbol{u}} \mathrm{d} \boldsymbol{x} \\
\quad=\int_{\Omega}\left(\frac{1}{\tilde{\varepsilon}_{0}}-\frac{1}{\tilde{\varepsilon}_{\rho_{n}}}\right) \operatorname{curl} \boldsymbol{H}_{0} \cdot \overline{\operatorname{curl} \boldsymbol{u}} \mathrm{d} \boldsymbol{x}-\omega^{2} \int_{\Omega}\left(\mu_{0}-\mu_{\rho_{n}}\right) \boldsymbol{H}_{0} \cdot \overline{\boldsymbol{u}} \mathrm{d} \boldsymbol{x}
\end{aligned}
$$

for all $\boldsymbol{u} \in \boldsymbol{H}(\operatorname{curl}, \Omega)$. The regularity results for weak solutions of Maxwell's equations mentioned before show that $\left\|\boldsymbol{H}_{0}\right\|_{\boldsymbol{W}^{1, \infty}\left(\Omega_{0}, \mathbb{C}^{3}\right)} \leq C\|\boldsymbol{g}\|_{\boldsymbol{H}_{\mathrm{div}}^{-1 / 2}(\partial \Omega)}$, and we may estimate

$$
\begin{aligned}
\mid \int_{\Omega}\left(\frac{1}{\tilde{\varepsilon}_{0}}-\frac{1}{\tilde{\varepsilon}_{\rho_{n}}}\right) \operatorname{curl} \boldsymbol{H}_{0} \cdot & \overline{\operatorname{curl} \boldsymbol{u}} \mathrm{d} \boldsymbol{x}-\omega^{2} \int_{\Omega}\left(\mu_{0}-\mu_{\rho_{n}}\right) \boldsymbol{H}_{0} \cdot \overline{\boldsymbol{u}} \mathrm{d} \boldsymbol{x} \mid \\
& \leq C\left|D_{\rho_{n}}\right|^{1 / 2}\left(\left\|\operatorname{curl} \boldsymbol{H}_{0}\right\|_{\boldsymbol{L}^{\infty}\left(\Omega_{0}, \mathbb{C}^{3}\right)}\|\operatorname{curl} \boldsymbol{u}\|_{\boldsymbol{L}^{2}\left(\Omega, \mathbb{C}^{3}\right)}+\left\|\boldsymbol{H}_{0}\right\|_{\boldsymbol{L}^{\infty}\left(\Omega_{0}, \mathbb{C}^{3}\right)}\|\boldsymbol{u}\|_{\boldsymbol{L}^{2}\left(\Omega, \mathbb{C}^{3}\right)}\right) \\
& \leq C\left|D_{\rho_{n}}\right|^{1 / 2}\|\boldsymbol{g}\|_{\boldsymbol{H}_{\mathrm{div}}^{-1 / 2}(\partial \Omega)}\|\boldsymbol{u}\|_{\boldsymbol{H}(\operatorname{curl}, \Omega)} .
\end{aligned}
$$

So, using orthogonality we obtain that $\boldsymbol{h}_{\rho_{n}}-\boldsymbol{h}_{0}^{\left(\rho_{n}\right)}$ satisfies

$$
a_{\rho_{n}}\left(\boldsymbol{h}_{\rho_{n}}-\boldsymbol{h}_{0}^{\left(\rho_{n}\right)}, \boldsymbol{v}\right)=l_{\rho_{n}}(\boldsymbol{v}) \quad \text { for all } \boldsymbol{v} \in \boldsymbol{Y}_{\rho_{n}}^{\perp},
$$

where $l_{\rho_{n}}$ is a bounded conjugate linear form on $\boldsymbol{Y}_{\rho_{n}}^{\perp}$ such that

$$
\sup _{\|\boldsymbol{v}\|_{\boldsymbol{H}(\mathbf{c u r l}, \Omega)}=1}\left|l_{\rho_{n}}(\boldsymbol{v})\right| \leq C\left|D_{\rho_{n}}\right|^{1 / 2}\|\boldsymbol{g}\|_{\boldsymbol{H}_{\mathrm{div}}^{-1 / 2}(\partial \Omega)} .
$$


Hence, (3.12a) is a consequence of the well-posedness of (3.14), which follows directly from the proof of [9], Lemma 1 recalling that we assumed $\left|D_{\rho_{n}}\right| \leq R$.

Step 2 (proof of (3.12b)). Since $\operatorname{div}\left(\mu_{0} \boldsymbol{h}_{0}\right)=0$ in $\Omega$ and $\mu_{0} \boldsymbol{\nu} \cdot \boldsymbol{h}_{0}=0$ on $\partial \Omega$ there exists a vector potential $\boldsymbol{z}_{0} \in$ $\boldsymbol{H}_{0}(\mathbf{c u r l}, \Omega)$ satisfying $\operatorname{div}\left(\mu_{0} \boldsymbol{z}_{0}\right)=0$ in $\Omega$ such that $\mu_{0} \boldsymbol{h}_{0}=\operatorname{curl} \boldsymbol{z}_{0}$ (cf. [37], Thm. 3.41). Moreover (see [37], Thm. 3.38), $\boldsymbol{z}_{0}=\operatorname{curl}(\boldsymbol{A})$ for some $\boldsymbol{A} \in \boldsymbol{H}(\operatorname{curl}, \Omega)$, which means $\boldsymbol{z}_{0} \in \operatorname{curl}(\boldsymbol{H}(\operatorname{curl}, \Omega))$. Analogously, we can find $\boldsymbol{z}_{0}^{\left(\rho_{n}\right)} \in \operatorname{curl}(\boldsymbol{H}(\operatorname{curl}, \Omega))$ such that $\mu_{\rho_{n}} \boldsymbol{h}_{0}^{\left(\rho_{n}\right)}=\operatorname{curl} \boldsymbol{z}_{0}^{\left(\rho_{n}\right)}$. Now, following an idea used in [37], p. 173, where it was attributed to Arnold et al. [12], we introduce sesquilinear forms $\alpha$ on $\boldsymbol{H}(\mathbf{c u r l}, \Omega) \times \boldsymbol{H}(\mathbf{c u r l}, \Omega)$ and $\beta$ on $\operatorname{curl}(\boldsymbol{H}(\operatorname{curl}, \Omega)) \times \boldsymbol{H}(\operatorname{curl}, \Omega)$ by

$$
\alpha(\boldsymbol{u}, \boldsymbol{v}):=\int_{\Omega} \mu_{\rho_{n}} \boldsymbol{u} \cdot \overline{\boldsymbol{v}} \mathrm{d} \boldsymbol{x} \quad \text { and } \quad \beta(\boldsymbol{w}, \boldsymbol{v}):=-\int_{\Omega} \boldsymbol{w} \cdot \overline{\operatorname{curl} \boldsymbol{v}} \mathrm{d} \boldsymbol{x},
$$

respectively, and observe that $\left(\boldsymbol{h}_{0}-\boldsymbol{h}_{0}^{\left(\rho_{n}\right)}, \boldsymbol{z}_{0}-\boldsymbol{z}_{0}^{\left(\rho_{n}\right)}\right)$ solves the mixed variational problem

$$
\begin{aligned}
\alpha\left(\boldsymbol{h}_{0}-\boldsymbol{h}_{0}^{\left(\rho_{n}\right)}, \boldsymbol{v}\right)+ & \beta\left(\boldsymbol{z}_{0}-\boldsymbol{z}_{0}^{\left(\rho_{n}\right)}, \boldsymbol{v}\right)=\int_{\Omega}\left(\mu_{\rho_{n}}-\mu_{0}\right) \boldsymbol{h}_{0} \cdot \overline{\boldsymbol{v}} \mathrm{d} \boldsymbol{x}, \\
\beta\left(\boldsymbol{w}, \boldsymbol{h}_{0}-\boldsymbol{h}_{0}^{\left(\rho_{n}\right)}\right) & =0
\end{aligned}
$$

for all $\boldsymbol{v} \in \boldsymbol{H}(\operatorname{curl}, \Omega)$ and $\boldsymbol{w} \in \operatorname{curl}(\boldsymbol{H}(\operatorname{curl}, \Omega))$. Note that $\alpha$ and $\beta$ are bounded, $\alpha$ is coercive, and $\beta$ satisfies the Babuška-Brezzi condition

$$
\sup _{\boldsymbol{v} \in \boldsymbol{H}(\operatorname{curl}, \Omega)} \frac{|\beta(\boldsymbol{w}, \boldsymbol{v})|}{\|\boldsymbol{v}\|_{\boldsymbol{H}(\operatorname{curl}, \Omega)}}=\sup _{\boldsymbol{v} \in \boldsymbol{H}(\operatorname{curl}, \Omega)} \frac{\left|\int_{\Omega} \boldsymbol{w} \cdot \overline{\operatorname{curl} \boldsymbol{v}} \mathrm{d} \boldsymbol{x}\right|}{\|\boldsymbol{v}\|_{\boldsymbol{H}(\operatorname{curl}, \Omega)}} \geq \frac{\|\boldsymbol{w}\|_{\boldsymbol{L}^{2}\left(\Omega, \mathbb{C}^{3}\right)}^{2}}{\|\boldsymbol{u}\|_{\boldsymbol{H}(\operatorname{curl}, \Omega)}} \geq C\|\boldsymbol{w}\|_{\boldsymbol{L}^{2}\left(\Omega, \mathbb{C}^{3}\right)}
$$

for all $\boldsymbol{w} \in \operatorname{curl}(\boldsymbol{H}(\operatorname{curl}, \Omega))$, where $\boldsymbol{u} \in \boldsymbol{Y}_{0}^{\perp} \subset \boldsymbol{H}(\operatorname{curl}, \Omega)$ has been chosen such that $\boldsymbol{w}=\operatorname{curl} \boldsymbol{u}$, and we used (3.3) to estimate $\|\boldsymbol{u}\|_{\boldsymbol{L}^{2}\left(\Omega, \mathbb{C}^{3}\right)} \leq C\|\boldsymbol{w}\|_{\boldsymbol{L}^{2}\left(\Omega, \mathbb{C}^{3}\right)}$. Therefore (cf. [37], Thm. 2.25), solutions to (3.15) are unique and $\left(\boldsymbol{h}_{0}-\boldsymbol{h}_{0}^{\left(\rho_{n}\right)}, \boldsymbol{z}_{0}-\boldsymbol{z}_{0}^{\left(\rho_{n}\right)}\right)$ satisfies

$$
\begin{aligned}
\left\|\boldsymbol{h}_{0}-\boldsymbol{h}_{0}^{\left(\rho_{n}\right)}\right\|_{\boldsymbol{H}(\operatorname{curl}, \Omega)}+\left\|\boldsymbol{z}_{0}-\boldsymbol{z}_{0}^{\left(\rho_{n}\right)}\right\|_{\boldsymbol{L}^{2}\left(\Omega, \mathbb{C}^{3}\right)} & \leq \sup _{\boldsymbol{u} \in \boldsymbol{H}(\operatorname{curl}, \Omega)} \frac{\left|\int_{\Omega}\left(\mu_{\rho_{n}}-\mu_{0}\right) \boldsymbol{h}_{0} \cdot \overline{\boldsymbol{u}} \mathrm{d} \boldsymbol{x}\right|}{\|\boldsymbol{u}\|_{\boldsymbol{H}(\operatorname{curl}, \Omega)}} \\
& \leq C\left|D_{\rho_{n}}\right|^{1 / 2}\left\|\boldsymbol{h}_{0}\right\|_{\boldsymbol{L}^{\infty}\left(\Omega_{0}, \mathbb{C}^{3}\right)} \leq C\left|D_{\rho_{n}}\right|^{1 / 2}\|\boldsymbol{g}\|_{\boldsymbol{H}_{\mathrm{div}}^{-1 / 2}(\partial \Omega)} .
\end{aligned}
$$

The last inequality follows from the definition of $\boldsymbol{h}_{0}=\boldsymbol{H}_{0}-\nabla q_{0}$ and the interior regularity of $q_{0}$ and $\boldsymbol{H}_{0}$ mentioned before.

Step 3 (proof of (3.13)). Similar to Step 1 we find by subtracting the weak formulations (2.3) and (2.4) that for all $\boldsymbol{u} \in \boldsymbol{H}(\operatorname{curl}, \Omega)$,

$$
\begin{aligned}
\int_{\Omega} \frac{1}{\tilde{\varepsilon}_{0}} \operatorname{curl}\left(\boldsymbol{H}_{\rho_{n}}-\boldsymbol{H}_{0}\right) \cdot \overline{\operatorname{curl} \boldsymbol{u}} \mathrm{d} \boldsymbol{x} & -\omega^{2} \int_{\Omega} \mu_{0}\left(\boldsymbol{H}_{\rho_{n}}-\boldsymbol{H}_{0}\right) \cdot \overline{\boldsymbol{u}} \mathrm{d} \boldsymbol{x} \\
& =\int_{\Omega}\left(\frac{1}{\tilde{\varepsilon}_{0}}-\frac{1}{\tilde{\varepsilon}_{\rho_{n}}}\right) \operatorname{curl} \boldsymbol{H}_{\rho_{n}} \cdot \overline{\operatorname{curl} \boldsymbol{u}} \mathrm{d} \boldsymbol{x}-\omega^{2} \int_{\Omega}\left(\mu_{0}-\mu_{\rho_{n}}\right) \boldsymbol{H}_{\rho_{n}} \cdot \overline{\boldsymbol{u}} \mathrm{d} \boldsymbol{x} .
\end{aligned}
$$

Using a duality argument inspired by the proof of [18], Lemma 1 we denote by $\boldsymbol{z} \in \boldsymbol{Y}_{0}^{\perp}$ the solution of the adjoint problem

$$
\int_{\Omega} \overline{\frac{1}{\tilde{\varepsilon}_{0}}} \operatorname{curl} \boldsymbol{z} \cdot \overline{\operatorname{curl} \boldsymbol{u}} \mathrm{d} \boldsymbol{x}-\omega^{2} \int_{\Omega} \mu_{0} \boldsymbol{z} \cdot \overline{\boldsymbol{u}} \mathrm{d} \boldsymbol{x}=\int_{\Omega} \mu_{0}\left(\boldsymbol{h}_{\rho_{n}}^{(0)}-\boldsymbol{h}_{0}\right) \cdot \overline{\boldsymbol{u}} \mathrm{d} \boldsymbol{x}
$$

for all $\boldsymbol{u} \in \boldsymbol{Y}_{0}^{\perp}$. Uniqueness of solutions to (2.3) implies existence and uniqueness of a solution to (3.17) with

$$
\|\boldsymbol{z}\|_{\boldsymbol{H}(\operatorname{curl}, \Omega)} \leq C\left\|\boldsymbol{h}_{\rho_{n}}^{(0)}-\boldsymbol{h}_{0}\right\|_{\boldsymbol{L}^{2}\left(\Omega, \mathbb{C}^{3}\right)} .
$$


Recalling that in $\boldsymbol{Y}_{0}^{\perp}$ the norm $\|\cdot\|_{\boldsymbol{H}^{1}\left(\Omega, \mathbb{C}^{3}\right)}$ is equivalent to $\|\cdot\|_{\boldsymbol{H}(\operatorname{curl}, \Omega)}$ (cf. Dautray and Lions [25], Thm. 3, p. 209), this shows that $\|\boldsymbol{z}\|_{\boldsymbol{H}^{1}\left(\Omega, \mathbb{C}^{3}\right)} \leq C\left\|\boldsymbol{h}_{\rho_{n}}^{(0)}-\boldsymbol{h}_{0}\right\|_{\boldsymbol{L}^{2}\left(\Omega, \mathbb{C}^{3}\right)}$. Similarly, since $\operatorname{curl} \boldsymbol{z}$ satisfies $\operatorname{curl}\left(\left(\overline{1 / \tilde{\varepsilon}_{0}}\right) \operatorname{curl} \boldsymbol{z}\right)=$ $\mu_{0}\left(\omega^{2} \boldsymbol{z}+\boldsymbol{h}_{\rho_{n}}-\boldsymbol{h}_{0}^{\left(\rho_{n}\right)}\right)$, i.e., $\operatorname{curl} \boldsymbol{z} \in \boldsymbol{H}(\operatorname{curl}, \Omega), \operatorname{div}(\operatorname{curl}(\boldsymbol{z}))=0$ in $\Omega$, and $\boldsymbol{\nu} \times \operatorname{curl} \boldsymbol{z}=0$ on $\partial \Omega$, we find that $\operatorname{curl} \boldsymbol{z} \in \boldsymbol{H}^{1}\left(\Omega, \mathbb{C}^{3}\right)$ and $\|\operatorname{curl} \boldsymbol{z}\|_{\boldsymbol{H}^{1}\left(\Omega, \mathbb{C}^{3}\right)} \leq C\left\|\boldsymbol{h}_{\rho_{n}}^{(0)}-\boldsymbol{h}_{0}\right\|_{\boldsymbol{L}^{2}\left(\Omega, \mathbb{C}^{3}\right)}$ as well. Substituting this $\boldsymbol{z}$ into (3.16), using orthogonality, (3.17), and Hölder's inequality we obtain that

$$
\begin{aligned}
\int_{\Omega}\left|\boldsymbol{h}_{\rho_{n}}^{(0)}-\boldsymbol{h}_{0}\right|^{2} \mathrm{~d} \boldsymbol{x} & =\left|\int_{\Omega} \frac{1}{\tilde{\varepsilon}_{0}} \operatorname{curl}\left(\boldsymbol{h}_{\rho_{n}}^{(0)}-\boldsymbol{h}_{0}\right) \cdot \overline{\operatorname{curl} \boldsymbol{z}} \mathrm{d} \boldsymbol{x}-\omega^{2} \int_{\Omega} \mu_{0}\left(\boldsymbol{h}_{\rho_{n}}^{(0)}-\boldsymbol{h}_{0}\right) \cdot \overline{\boldsymbol{z}} \mathrm{d} \boldsymbol{x}\right| \\
& =\left|\int_{\Omega}\left(\frac{1}{\tilde{\varepsilon}_{0}}-\frac{1}{\varepsilon_{\rho_{n}}}\right) \operatorname{curl} \boldsymbol{H}_{\rho_{n}} \cdot \overline{\operatorname{curl} \boldsymbol{z}} \mathrm{d} \boldsymbol{x}-\omega^{2} \int_{\Omega}\left(\mu_{0}-\mu_{\rho_{n}}\right) \boldsymbol{H}_{\rho_{n}} \cdot \overline{\boldsymbol{z}} \mathrm{d} \boldsymbol{x}\right| \\
& \leq C\left(\left\|\operatorname{curl} \boldsymbol{H}_{\rho_{n}}\right\|_{\boldsymbol{L}^{q}\left(D_{\rho_{n}}, \mathbb{C}^{3}\right)}\|\operatorname{curl} \boldsymbol{z}\|_{\boldsymbol{L}^{p}\left(D_{\rho_{n}}, \mathbb{C}^{3}\right)}+\left\|\boldsymbol{H}_{\rho_{n}}\right\|_{\boldsymbol{L}^{q}\left(D_{\rho_{n}}, \mathbb{C}^{3}\right)}\|\boldsymbol{z}\|_{\boldsymbol{L}^{p}\left(D_{\rho_{n}}, \mathbb{C}^{3}\right)}\right),
\end{aligned}
$$

where $1 \leq p, q \leq \infty$ are such that $1 / p+1 / q=1$. Assuming that $2 \leq p \leq 6$, i.e., $6 / 5 \leq q \leq 2$, the boundedness of the embedding of $\boldsymbol{H}^{1}\left(\Omega, \mathbb{C}^{3}\right)$ into $\boldsymbol{L}^{p}\left(\Omega, \mathbb{C}^{3}\right)$ (cf. Adams [1], Thm. 5.4) implies that

$$
\|\operatorname{curl} \boldsymbol{z}\|_{\boldsymbol{L}^{p}\left(D_{\rho_{n}}, \mathbb{C}^{3}\right)} \leq C\|\operatorname{curl} \boldsymbol{z}\|_{\boldsymbol{H}^{1}\left(\Omega, \mathbb{C}^{3}\right)} \quad \text { and } \quad\|\boldsymbol{z}\|_{\boldsymbol{L}^{p}\left(D_{\rho_{n}}, \mathbb{C}^{3}\right)} \leq C\|\boldsymbol{z}\|_{\boldsymbol{H}^{1}\left(\Omega, \mathbb{C}^{3}\right)} .
$$

Thus,

$$
\left\|\boldsymbol{h}_{\rho_{n}}^{(0)}-\boldsymbol{h}_{0}\right\|_{\boldsymbol{L}^{2}\left(\Omega, \mathbb{C}^{3}\right)} \leq C\left(\left\|\operatorname{curl} \boldsymbol{H}_{\rho_{n}}\right\|_{\boldsymbol{L}^{q}\left(D_{\rho_{n}}, \mathbb{C}^{3}\right)}+\left\|\boldsymbol{H}_{\rho_{n}}\right\|_{\boldsymbol{L}^{q}\left(D_{\rho_{n}}, \mathbb{C}^{3}\right)}\right) .
$$

Since 6/5 $\leq q \leq 2$, we can use the triangle inequality and embed $\boldsymbol{L}^{2}\left(D_{\rho_{n}}, \mathbb{C}^{3}\right)$ and $\boldsymbol{L}^{\infty}\left(D_{\rho_{n}}, \mathbb{C}^{3}\right)$ into $\boldsymbol{L}^{q}\left(D_{\rho_{n}}, \mathbb{C}^{3}\right)$ applying Hölder's inequality (cf. Gilbarg and Trudinger [27], Eq. (7.8), p. 146) to see that

$$
\left\|\boldsymbol{H}_{\rho_{n}}\right\|_{\boldsymbol{L}^{q}\left(D_{\rho_{n}}, \mathbb{C}^{3}\right)}+\left\|\operatorname{curl} \boldsymbol{H}_{\rho_{n}}\right\|_{\boldsymbol{L}^{q}\left(D_{\rho_{n}}, \mathbb{C}^{3}\right)} \leq\left|D_{\rho_{n}}\right|^{1 / q-1 / 2}\left\|\boldsymbol{H}_{\rho_{n}}-\boldsymbol{H}_{0}\right\|_{\boldsymbol{H}(\operatorname{curl}, \Omega)}+\left|D_{\rho_{n}}\right|^{1 / q}\left\|\boldsymbol{H}_{0}\right\|_{\boldsymbol{W}^{1, \infty}\left(\Omega_{0}, \mathbb{C}^{3}\right)} .
$$

Recalling that $\left\|\boldsymbol{H}_{0}\right\|_{\boldsymbol{W}^{1, \infty}\left(\Omega_{0}, \mathbb{C}^{3}\right)} \leq C\|\boldsymbol{g}\|_{\boldsymbol{H}_{\mathrm{div}}^{-1 / 2}(\partial \Omega)}$, we use (3.4) to obtain

$$
\left\|\boldsymbol{H}_{\rho_{n}}-\boldsymbol{H}_{0}\right\|_{\boldsymbol{H}(\mathbf{c u r l}, \Omega)} \leq\left\|\boldsymbol{h}_{\rho_{n}}-\boldsymbol{h}_{0}\right\|_{\boldsymbol{H}(\mathbf{c u r l}, \Omega)}+\left\|q_{\rho_{n}}-q_{0}\right\|_{H^{1}(\Omega, \mathbb{C})},
$$

which together with (3.10), (3.12a), and (3.12b) gives

$$
\left\|\boldsymbol{H}_{\rho_{n}}-\boldsymbol{H}_{0}\right\|_{\boldsymbol{H}(\mathbf{c u r l}, \Omega)} \leq C\left|D_{\rho_{n}}\right|^{1 / 2}\|\boldsymbol{g}\|_{\boldsymbol{H}_{\mathrm{div}}^{-1 / 2}(\partial \Omega)} .
$$

Therefore, combining (3.19) and (3.18) we find that

$$
\left\|\boldsymbol{h}_{\rho_{n}}^{(0)}-\boldsymbol{h}_{0}\right\|_{\boldsymbol{L}^{2}\left(\Omega, \mathbb{C}^{3}\right)} \leq C\left|D_{\rho_{n}}\right|^{1 / q}\|\boldsymbol{g}\|_{\boldsymbol{H}_{\mathrm{div}}^{-1 / 2}(\partial \Omega)}
$$

and writing $\eta:=1-1 / q$ yields (3.13).

Step 4 (proof of (3.12c)). As in Step 2 we can find $\boldsymbol{z}_{\rho_{n}}, \boldsymbol{z}_{\rho_{n}}^{(0)} \in \operatorname{curl}(\boldsymbol{H}(\operatorname{curl}, \Omega))$ such that $\mu_{\rho_{n}} \boldsymbol{h}_{\rho_{n}}=\operatorname{curl} \boldsymbol{z}_{\rho_{n}}$ and $\mu_{0} \boldsymbol{h}_{\rho_{n}}^{(0)}=\operatorname{curl} \boldsymbol{z}_{\rho_{n}}^{(0)}$. Then $\left(\boldsymbol{h}_{\rho_{n}}-\boldsymbol{h}_{\rho_{n}}^{(0)}, \boldsymbol{z}_{\rho_{n}}-\boldsymbol{z}_{\rho_{n}}^{(0)}\right)$ satisfies

$$
\begin{aligned}
\alpha\left(\boldsymbol{h}_{\rho_{n}}-\boldsymbol{h}_{\rho_{n}}^{(0)}, \boldsymbol{v}\right)+\beta\left(\boldsymbol{z}_{\rho_{n}}-\boldsymbol{z}_{\rho_{n}}^{(0)}, \boldsymbol{v}\right) & =\int_{\Omega}\left(\mu_{0}-\mu_{\rho_{n}}\right) \boldsymbol{h}_{\rho_{n}}^{(0)} \cdot \overline{\boldsymbol{v}} \mathrm{d} \boldsymbol{x}, \\
\beta\left(\boldsymbol{w}, \boldsymbol{h}_{\rho_{n}}-\boldsymbol{h}_{\rho_{n}}^{(0)}\right) & =0
\end{aligned}
$$


for all $\boldsymbol{v} \in \boldsymbol{H}(\operatorname{curl}, \Omega)$ and $\boldsymbol{w} \in \operatorname{curl}(\boldsymbol{H}(\operatorname{curl}, \Omega))$, where $\alpha$ and $\beta$ are the sesquilinear forms introduced in the second step. Therefore, using (3.13) we obtain that

$$
\begin{aligned}
\left\|\boldsymbol{h}_{\rho_{n}}-\boldsymbol{h}_{\rho_{n}}^{(0)}\right\|_{\boldsymbol{H}(\operatorname{curl}, \Omega)}+\left\|\boldsymbol{z}_{\rho_{n}}-\boldsymbol{z}_{\rho_{n}}^{(0)}\right\|_{\boldsymbol{L}^{2}\left(\Omega, \mathbb{C}^{3}\right)} & \leq\left\|\left(\mu_{0}-\mu_{\rho_{n}}\right) \boldsymbol{h}_{\rho_{n}}^{(0)}\right\|_{\boldsymbol{L}^{2}\left(\Omega, \mathbb{C}^{3}\right)} \\
& \leq\left\|\left(\mu_{0}-\mu_{\rho_{n}}\right) \boldsymbol{h}_{0}\right\|_{\boldsymbol{L}^{2}\left(\Omega, \mathbb{C}^{3}\right)}+\left\|\left(\mu_{0}-\mu_{\rho_{n}}\right)\left(\boldsymbol{h}_{0}-\boldsymbol{h}_{\rho_{n}}^{(0)}\right)\right\|_{\boldsymbol{L}^{2}\left(\Omega, \mathbb{C}^{3}\right)} \\
& \leq C\left|D_{\rho_{n}}\right|^{1 / 2}\left\|\boldsymbol{h}_{0}\right\|_{\boldsymbol{L}^{\infty}\left(\Omega_{0}, \mathbb{C}^{3}\right)}+C_{\eta}\left|D_{\rho_{n}}\right|^{1-\eta}\|\boldsymbol{g}\|_{\boldsymbol{H}_{\mathrm{div}}}^{-1 / 2}(\partial \Omega) \\
& \leq C\left|D_{\rho_{n}}\right|^{1 / 2}\|\boldsymbol{g}\|_{\boldsymbol{H}_{\mathrm{div}}^{-1 / 2}(\partial \Omega)} .
\end{aligned}
$$

Lemma 3.3. Let $q_{0}, q_{0}^{\left(\rho_{n}\right)}, q_{\rho_{n}}$, and $q_{\rho_{n}}^{(0)}$ be as in (3.4) and (3.7), respectively. Then, there exists a constant $C$ such that for any $n \in \mathbb{N}$,

$$
\begin{aligned}
\left\|q_{0}-q_{0}^{\left(\rho_{n}\right)}\right\|_{H^{1}(\Omega, \mathbb{C})} & \leq C\left|D_{\rho_{n}}\right|^{1 / 2}\|\boldsymbol{g}\|_{\boldsymbol{H}_{\mathrm{div}}^{-1 / 2}(\partial \Omega)}, \\
\left\|q_{\rho_{n}}-q_{\rho_{n}}^{(0)}\right\|_{H^{1}(\Omega, \mathbb{C})} & \leq C\left|D_{\rho_{n}}\right|^{1 / 2}\|\boldsymbol{g}\|_{\boldsymbol{H}_{\mathrm{div}}^{-1 / 2}(\partial \Omega)} .
\end{aligned}
$$

Furthermore, for any $\eta \in[1 / 5,1 / 2]$ there exists a constant $C_{\eta}$ such that

$$
\begin{aligned}
\left\|q_{0}-q_{0}^{\left(\rho_{n}\right)}\right\|_{L^{2}(\Omega, \mathbb{C})} \leq C_{\eta}\left|D_{\rho_{n}}\right|^{1-\eta}\|\boldsymbol{g}\|_{\boldsymbol{H}_{\mathrm{div}}^{-1 / 2}(\partial \Omega)}, \\
\left\|q_{\rho_{n}}-q_{\rho_{n}}^{(0)}\right\|_{L^{2}(\Omega, \mathbb{C})} \leq C_{\eta}\left|D_{\rho_{n}}\right|^{1-\eta}\|\boldsymbol{g}\|_{\boldsymbol{H}_{\mathrm{div}}^{-1 / 2}(\partial \Omega)}
\end{aligned}
$$

Proof. These estimates are no direct consequences of [18], Lemma 1 because the source terms in (3.8) and (3.9) are discontinuous and do not agree with the source terms in (3.5) and (3.6), respectively. However, the lemma can be shown following the same strategy as used in the proof of [18], Lemma 1. Therefore, we comment on differences to this proof only, without including all details.

Step 1 (proof of (3.20a)). From the weak formulations of (3.5) and (3.8) we find that

$$
\begin{aligned}
\int_{\Omega} \mu_{0} \nabla q_{0} \cdot \overline{\nabla \phi} \mathrm{d} \boldsymbol{x} & =\int_{\Omega} \mu_{0} \boldsymbol{H}_{0} \cdot \overline{\nabla \phi} \mathrm{d} \boldsymbol{x} & & \text { for all } \phi \in H^{1}(\Omega, \mathbb{C}), \\
\int_{\Omega} \mu_{\rho_{n}} \nabla q_{0}^{\left(\rho_{n}\right)} \cdot \overline{\nabla \phi} \mathrm{d} \boldsymbol{x} & =\int_{\Omega} \mu_{\rho_{n}} \boldsymbol{H}_{0} \cdot \overline{\nabla \phi} \mathrm{d} \boldsymbol{x} & & \text { for all } \phi \in H^{1}(\Omega, \mathbb{C}),
\end{aligned}
$$

and therefore

$$
\int_{\Omega} \mu_{\rho_{n}} \nabla\left(q_{0}-q_{0}^{\left(\rho_{n}\right)}\right) \cdot \overline{\nabla \phi} \mathrm{d} \boldsymbol{x}=\int_{\Omega}\left(\mu_{0}-\mu_{\rho_{n}}\right) \boldsymbol{h}_{0} \cdot \overline{\nabla \phi} \mathrm{d} \boldsymbol{x}
$$

for all $\phi \in H^{1}(\Omega, \mathbb{C})$. Using the smoothness of $\boldsymbol{h}_{0}$ in $\Omega_{0}$, which follows from the smoothness of $\boldsymbol{H}_{0}$ and $q_{0}$, we can proceed as in the proof of [18], Lemma 1 to obtain (3.20a).

Step 2 (proof of (3.20b)). Similar to the first step we find from (3.6) and (3.9) that

$$
\begin{array}{ll}
\int_{\Omega} \mu_{\rho_{n}} \nabla q_{\rho_{n}} \cdot \overline{\nabla \phi} \mathrm{d} \boldsymbol{x}=\int_{\Omega} \mu_{\rho_{n}} \boldsymbol{H}_{\rho_{n}} \cdot \overline{\nabla \phi} \mathrm{d} \boldsymbol{x} & \text { for all } \phi \in H^{1}(\Omega, \mathbb{C}), \\
\int_{\Omega} \mu_{0} \nabla q_{\rho_{n}}^{(0)} \cdot \overline{\nabla \phi} \mathrm{d} \boldsymbol{x}=\int_{\Omega} \mu_{0} \boldsymbol{H}_{\rho_{n}} \cdot \overline{\nabla \phi} \mathrm{d} \boldsymbol{x} & \text { for all } \phi \in H^{1}(\Omega, \mathbb{C}),
\end{array}
$$

and thus

$$
\begin{aligned}
\int_{\Omega} \mu_{\rho_{n}} \nabla\left(q_{\rho_{n}}-q_{\rho_{n}}^{(0)}\right) \cdot \overline{\nabla \phi} \mathrm{d} \boldsymbol{x} & =\int_{\Omega}\left(\mu_{\rho_{n}}-\mu_{0}\right) \boldsymbol{h}_{\rho_{n}}^{(0)} \cdot \overline{\nabla \phi} \mathrm{d} \boldsymbol{x} \\
& =\int_{\Omega}\left(\mu_{\rho_{n}}-\mu_{0}\right)\left(\boldsymbol{h}_{\rho_{n}}^{(0)}-\boldsymbol{h}_{0}\right) \cdot \overline{\nabla \phi} \mathrm{d} \boldsymbol{x}+\int_{\Omega}\left(\mu_{\rho_{n}}-\mu_{0}\right) \boldsymbol{h}_{0} \cdot \overline{\nabla \phi} \mathrm{d} \boldsymbol{x}
\end{aligned}
$$


for all $\phi \in H^{1}(\Omega, \mathbb{C})$. Using (3.13) and the smoothness of $\boldsymbol{h}_{0}$ in $\Omega_{0}$ mentioned before we estimate

$$
\begin{aligned}
\mid \int_{\Omega}\left(\mu_{\rho_{n}}-\mu_{0}\right)\left(\boldsymbol{h}_{\rho_{n}}^{(0)}-\boldsymbol{h}_{0}\right) \cdot \overline{\nabla \phi} \mathrm{d} \boldsymbol{x}+ & \int_{\Omega}\left(\mu_{\rho_{n}}-\mu_{0}\right) \boldsymbol{h}_{0} \cdot \overline{\nabla \phi} \mathrm{d} \boldsymbol{x} \mid \\
& \leq C\left\|\boldsymbol{h}_{\rho_{n}}^{(0)}-\boldsymbol{h}_{0}\right\|_{\boldsymbol{L}^{2}\left(\Omega, \mathbb{C}^{3}\right)}\|\phi\|_{H^{1}(\Omega, \mathbb{C})}+C\left|D_{\rho_{n}}\right|^{1 / 2}\left\|\boldsymbol{h}_{0}\right\|_{\boldsymbol{L}^{\infty}\left(\Omega_{0}, \mathbb{C}^{3}\right)}\|\phi\|_{H^{1}(\Omega, \mathbb{C})} \\
& \leq C\left|D_{\rho_{n}}\right|^{1 / 2}\|\boldsymbol{g}\|_{\boldsymbol{H}_{\mathrm{div}}^{-1 / 2}(\partial \Omega)}\|\phi\|_{H^{1}(\Omega, \mathbb{C})} .
\end{aligned}
$$

Therefore, $q_{\rho_{n}}-q_{\rho_{n}}^{(0)} \in H_{\diamond}^{1}(\Omega, \mathbb{C})$ satisfies

$$
\operatorname{div}\left(\mu_{\rho_{n}} \nabla\left(q_{\rho_{n}}-q_{\rho_{n}}^{(0)}\right)\right)=G_{\rho_{n}} \quad \text { in } \Omega, \quad \mu_{\rho_{n}} \frac{\partial\left(q_{\rho_{n}}-q_{\rho_{n}}^{(0)}\right)}{\partial \boldsymbol{\nu}}=0 \quad \text { on } \partial \Omega
$$

for some $G_{\rho_{n}} \in H^{-1}(\Omega, \mathbb{C})$ with $\left\|G_{\rho_{n}}\right\|_{H^{-1}(\Omega, \mathbb{C})} \leq C\left|D_{\rho_{n}}\right|^{1 / 2}\|\boldsymbol{g}\|_{\boldsymbol{H}_{\mathrm{div}}^{-1 / 2}(\partial \Omega)}$. This implies (3.20b).

Step 3 (proof of (3.21a)). From (3.22) we obtain that

$$
\int_{\Omega} \mu_{0} \nabla\left(q_{0}-q_{0}^{\left(\rho_{n}\right)}\right) \cdot \overline{\nabla \phi} \mathrm{d} \boldsymbol{x}=\int_{\Omega}\left(\mu_{0}-\mu_{\rho_{n}}\right) \boldsymbol{h}_{0}^{\left(\rho_{n}\right)} \cdot \overline{\nabla \phi} \mathrm{d} \boldsymbol{x} .
$$

Therewith (3.21a) follows using a duality argument as in the second part of the proof of [18], Lemma 1.

Step 4 (proof of $(3.21 \mathrm{~b})$ ). Using (3.23) we find that

$$
\int_{\Omega} \mu_{0} \nabla\left(q_{\rho_{n}}-q_{\rho_{n}}^{(0)}\right) \cdot \overline{\nabla \phi} \mathrm{d} \boldsymbol{x}=\int_{\Omega}\left(\mu_{\rho_{n}}-\mu_{0}\right) \boldsymbol{h}_{\rho_{n}} \cdot \overline{\nabla \phi} \mathrm{d} \boldsymbol{x} .
$$

Therewith (3.21b) can be shown using a duality argument as in the second part of the proof of [18], Lemma 1.

\subsection{Asymptotic correctors and polarization tensors}

In this section we introduce scalar-valued functions $v_{\mu_{0}}^{(j)}, v_{\mu_{\rho_{n}}}^{(j)}, 1 \leq j \leq 3$, vector-valued functions $\boldsymbol{w}_{\tilde{\varepsilon}_{0}}^{(j)}, \boldsymbol{w}_{\tilde{\varepsilon}_{\rho_{n}}}^{(j)}$, $1 \leq j \leq 3$, and the so-called electric and magnetic polarization tensors that will be used to derive the asymptotic perturbation formula in Section 4 .

For $1 \leq j \leq 3$, denote by $v_{\mu_{0}}^{(j)}, v_{\mu_{\rho_{n}}}^{(j)} \in H_{\diamond}^{1}(\Omega, \mathbb{C})$ the weak solutions of

$$
\begin{aligned}
\operatorname{div}\left(\mu_{0} \nabla v_{\mu_{0}}^{(j)}\right) & =\operatorname{div}\left(\mu_{0} \boldsymbol{e}_{j}\right) & & \text { in } \Omega, \\
\mu_{0} \frac{\partial v_{\mu_{0}}^{(j)}}{\partial \boldsymbol{\nu}} & =\mu_{0} \nu_{j} & & \text { on } \partial \Omega,
\end{aligned}
$$

and

$$
\begin{aligned}
\operatorname{div}\left(\mu_{\rho_{n}} \nabla v_{\mu_{\rho_{n}}}^{(j)}\right) & =\operatorname{div}\left(\mu_{0} \boldsymbol{e}_{j}\right) & & \text { in } \Omega, \\
\mu_{\rho_{n}} \frac{\partial v_{\mu_{\rho_{n}}}^{(j)}}{\partial \boldsymbol{\nu}} & =\mu_{0} \nu_{j} & & \text { on } \partial \Omega,
\end{aligned}
$$

in $H_{\diamond}^{1}(\Omega, \mathbb{C})$, respectively, where $\left(\boldsymbol{e}_{1}, \boldsymbol{e}_{2}, \boldsymbol{e}_{3}\right)$ is the standard basis of $\mathbb{R}^{3}$ and $\nu_{j}:=\boldsymbol{\nu} \cdot \boldsymbol{e}_{j}$ denotes the $j$ th component of the unit outward normal vector on $\partial \Omega$. Observe that $v_{\mu_{0}}^{(j)}$ is given by the explicit formula

$$
v_{\mu_{0}}^{(j)}=x_{j}-\frac{1}{|\partial \Omega|} \int_{\Omega} x_{j} \mathrm{~d} s(\boldsymbol{x})
$$


Lemma 3.4. Let $v_{\mu_{0}}^{(j)}$ and $v_{\mu_{\rho_{n}}}^{(j)}, 1 \leq j \leq 3$, be the solutions of (3.24) and (3.25), respectively. Then, there exists a constant $C$ such that for all $n \in \mathbb{N}$,

$$
\left\|v_{\mu_{\rho_{n}}}^{(j)}-v_{\mu_{0}}^{(j)}\right\|_{H^{1}(\Omega, \mathbb{C})} \leq C\left|D_{\rho_{n}}\right|^{1 / 2}
$$

and for any $\eta \in[1 / 5,1 / 2]$ there exists a constant $C_{\eta}$ such that

$$
\left\|v_{\mu_{\rho_{n}}}^{(j)}-v_{\mu_{0}}^{(j)}\right\|_{L^{2}(\Omega, \mathbb{C})} \leq C_{\eta}\left|D_{\rho_{n}}\right|^{1-\eta}
$$

Proof. This lemma is a direct consequence of [18], Lemma 1.

Lemma 3.5. For $1 \leq j \leq 3$ denote by $v_{\mu_{\rho_{n}}}^{(j)}, n \in \mathbb{N}$, the solution to (3.25). Then, there exists a subsequence, also denoted by $\left(D_{\rho_{n}}\right)_{n \in \mathbb{N}}$, and a matrix-valued function $\mathbb{M}^{\mu}:=\left(M_{i j}^{\mu}\right)_{1 \leq i, j \leq 3} \in \boldsymbol{L}^{2}\left(\Omega, \mathbb{R}^{3 \times 3} ; \mu\right)$, called magnetic polarization tensor, such that for $1 \leq i, j \leq 3$ and all $\phi \in C_{0}(\Omega, \mathbb{C})$,

$$
\int_{\Omega} \phi \frac{\partial v_{\mu_{\rho_{n}}}^{(j)}}{\partial x_{i}}\left|D_{\rho_{n}}\right|^{-1} \chi_{D_{\rho_{n}}} \mathrm{~d} \boldsymbol{x} \rightarrow \int_{\Omega} \phi M_{i j}^{\mu} \mathrm{d} \mu \quad \text { as } n \rightarrow \infty .
$$

Proof. This lemma has been shown in [18], pp. 165-166.

Remark 3.6. The definition of the magnetic polarization tensor $\mathbb{M}^{\mu}$ in (3.28) is equivalent to the definition of the polarization tensor appearing in the general perturbation formula for steady state voltage potentials established in [18]. This means that all results for polarization tensors obtained in [18,19,21] remain valid for the magnetic polarization tensor $\mathbb{M}^{\mu}$; in particular $(2.8 \mathrm{a})$ and $(2.8 \mathrm{~b})$ hold.

Next, for $1 \leq j \leq 3$ denote by $\boldsymbol{w}_{\tilde{\varepsilon}_{0}}^{(j)} \in \boldsymbol{Y}_{0}^{\perp}$ and $\boldsymbol{w}_{\tilde{\varepsilon}_{\rho_{n}}}^{(j)} \in \boldsymbol{Y}_{\rho_{n}}^{\perp}$ the weak solutions of

$$
\begin{array}{cc}
\operatorname{curl}\left(\frac{1}{\tilde{\varepsilon}_{0}} \operatorname{curl} \boldsymbol{w}_{\tilde{\varepsilon}_{0}}^{(j)}\right)=\operatorname{curl}\left(\frac{1}{\tilde{\varepsilon}_{0}} e_{j}\right) & \text { in } \Omega \\
\frac{1}{\tilde{\varepsilon}_{0}} \operatorname{curl} \boldsymbol{w}_{\tilde{\varepsilon}_{0}}^{(j)} \times \nu=\frac{1}{\tilde{\varepsilon}_{0}} \boldsymbol{e}_{j} \times \nu & \text { on } \partial \Omega
\end{array}
$$

and

$$
\begin{array}{cl}
\operatorname{curl}\left(\frac{1}{\tilde{\varepsilon}_{\rho_{n}}} \operatorname{curl} \boldsymbol{w}_{\tilde{\varepsilon}_{\rho_{n}}}^{(j)}\right)=\operatorname{curl}\left(\frac{1}{\tilde{\varepsilon}_{0}} e_{j}\right) & \text { in } \Omega \\
\frac{1}{\tilde{\varepsilon}_{\rho_{n}}} \operatorname{curl} \boldsymbol{w}_{\tilde{\varepsilon}_{\rho_{n}}}^{(j)} \times \nu=\frac{1}{\tilde{\varepsilon}_{0}} \boldsymbol{e}_{j} \times \nu & \text { on } \partial \Omega,
\end{array}
$$

respectively. Introducing the sesquilinear forms

$$
\begin{array}{ll}
a_{0}^{\text {stat }}(\boldsymbol{u}, \boldsymbol{v}):=\int_{\Omega} \frac{1}{\tilde{\varepsilon}_{0}} \operatorname{curl} \boldsymbol{u} \cdot \overline{\operatorname{curl} \boldsymbol{v}} \mathrm{d} \boldsymbol{x}, & \boldsymbol{u}, \boldsymbol{v} \in \boldsymbol{H}(\operatorname{curl}, \Omega), \\
a_{\rho_{n}}^{\text {stat }}(\boldsymbol{u}, \boldsymbol{v}):=\int_{\Omega} \frac{1}{\tilde{\varepsilon}_{\rho_{n}}} \operatorname{curl} \boldsymbol{u} \cdot \overline{\operatorname{curl} \boldsymbol{v}} \mathrm{d} \boldsymbol{x}, & \boldsymbol{u}, \boldsymbol{v} \in \boldsymbol{H}(\operatorname{curl}, \Omega),
\end{array}
$$

and the conjugate linear form

$$
l_{w}(\boldsymbol{v}):=\int_{\partial \Omega} \frac{1}{\tilde{\varepsilon}_{0}}\left(\boldsymbol{e}_{j} \times \boldsymbol{\nu}\right) \cdot \overline{\boldsymbol{v}} \mathrm{d} s, \quad \boldsymbol{v} \in \boldsymbol{H}(\operatorname{curl}, \Omega),
$$


the weak formulations of (3.29) and (3.30) ask to find $\boldsymbol{w}_{\tilde{\varepsilon}_{0}}^{(j)} \in \boldsymbol{Y}_{0}^{\perp}$ and $\boldsymbol{w}_{\tilde{\varepsilon}_{\rho_{n}}}^{(j)} \in \boldsymbol{Y}_{\rho_{n}}^{\perp}$ such that

$$
\begin{aligned}
a_{0}^{\text {stat }}\left(\boldsymbol{w}_{\tilde{\varepsilon}_{0}}^{(j)}, \boldsymbol{v}\right)=l_{w}(\boldsymbol{v}) & \text { for all } \boldsymbol{v} \in \boldsymbol{Y}_{0}^{\perp}, \\
a_{\rho_{n}}^{\text {stat }}\left(\boldsymbol{w}_{\tilde{\varepsilon}_{\rho_{n}}}^{(j)}, \boldsymbol{v}\right)=l_{w}(\boldsymbol{v}) & \text { for all } \boldsymbol{v} \in \boldsymbol{Y}_{\rho_{n}}^{\perp}
\end{aligned}
$$

Note that both equations are trivially fulfilled for any $\boldsymbol{v} \in \boldsymbol{Y}$. The sesquilinear forms $a_{0}^{\text {stat }}$ and $a_{\rho_{n}}^{\text {stat }}$ are bounded and recalling (3.3) we find that they are also coercive on $\boldsymbol{Y}_{0}^{\perp}$ as well as on $\boldsymbol{Y}_{\rho_{n}}^{\perp}$. Thus we obtain existence and uniqueness of solutions $\boldsymbol{w}_{0}^{(j)} \in \boldsymbol{Y}_{0}^{\perp}$ and $\boldsymbol{w}_{\rho_{n}}^{(j)} \in \boldsymbol{Y}_{\rho_{n}}^{\perp}$ from the Lax-Milgram Lemma and these solutions satisfy

$$
\left\|\boldsymbol{w}_{\tilde{\varepsilon}_{0}}^{(j)}\right\|_{\boldsymbol{H}(\operatorname{curl}, \Omega)} \leq C \quad \text { and } \quad\left\|\boldsymbol{w}_{\tilde{\varepsilon}_{\rho_{n}}}^{(j)}\right\|_{\boldsymbol{H}(\operatorname{curl}, \Omega)} \leq C,
$$

where $C$ is independent of $\rho_{n}$. In addition we define

$$
\boldsymbol{w}_{\tilde{\varepsilon}_{0}}^{\left(j, \rho_{n}\right)}:=P_{\rho_{n}} \boldsymbol{w}_{\tilde{\varepsilon}_{0}}^{(j)} \in \boldsymbol{Y}_{\rho_{n}}^{\perp} \quad \text { and } \quad \boldsymbol{w}_{\tilde{\varepsilon}_{\rho_{n}}}^{(j, 0)}:=P_{0} \boldsymbol{w}_{\tilde{\varepsilon}_{\rho_{n}}}^{(j)} \in \boldsymbol{Y}_{0}^{\perp},
$$

where $P_{\rho_{n}}$ and $P_{0}$ are the projection operators from (3.1). Observe that these function solve

$$
\begin{aligned}
a_{0}^{\text {stat }}\left(\boldsymbol{w}_{\tilde{\varepsilon}_{0}}^{\left(j, \rho_{n}\right)}, \boldsymbol{v}\right) & =l_{w}(\boldsymbol{v}) & & \text { for all } \boldsymbol{v} \in \boldsymbol{Y}_{\rho_{n}}^{\perp}, \\
a_{\rho_{n}}^{\text {stat }}\left(\boldsymbol{w}_{\tilde{\varepsilon}_{\rho_{n}}}^{(j, 0)}, \boldsymbol{v}\right) & =l_{w}(\boldsymbol{v}) & & \text { for all } \boldsymbol{v} \in \boldsymbol{Y}_{0}^{\perp},
\end{aligned}
$$

respectively, and therefore,

$$
\left\|\boldsymbol{w}_{\tilde{\varepsilon}_{0}}^{\left(j, \rho_{n}\right)}\right\|_{\boldsymbol{H}(\operatorname{curl}, \Omega)} \leq C \quad \text { and } \quad\left\|\boldsymbol{w}_{\tilde{\varepsilon}_{\rho_{n}}}^{(j, 0)}\right\|_{\boldsymbol{H}(\operatorname{curl}, \Omega)} \leq C,
$$

where $C$ is again independent of $\rho_{n}$.

Lemma 3.7. Let $\boldsymbol{w}_{\tilde{\varepsilon}_{0}}^{(j)}, \boldsymbol{w}_{\tilde{\varepsilon}_{0}}^{\left(j, \rho_{n}\right)}, \boldsymbol{w}_{\tilde{\varepsilon}_{\rho_{n}}}^{(j)}$, and $\boldsymbol{w}_{\tilde{\varepsilon}_{\rho_{n}}}^{(j, 0)}$ denote the solutions of (3.32) and (3.33), respectively. Then, there exists a constant $C$ such that for any $n \in \mathbb{N}$,

$$
\begin{aligned}
\left\|\boldsymbol{w}_{\tilde{\varepsilon}_{\rho_{n}}}^{(j)}-\boldsymbol{w}_{\tilde{\varepsilon}_{0}}^{\left(j, \rho_{n}\right)}\right\|_{\boldsymbol{H}(\operatorname{curl}, \Omega)} & \leq C\left|D_{\rho_{n}}\right|^{1 / 2}, \\
\left\|\boldsymbol{w}_{\tilde{\varepsilon}_{0}}^{(j)}-\boldsymbol{w}_{\tilde{\varepsilon}_{0}}^{\left(j, \rho_{n}\right)}\right\|_{\boldsymbol{H}(\operatorname{curl}, \Omega)} & \leq C\left|D_{\rho_{n}}\right|^{1 / 2}, \\
\left\|\boldsymbol{w}_{\tilde{\varepsilon}_{\rho_{n}}}^{(j)}-\boldsymbol{w}_{\tilde{\varepsilon}_{\rho_{n}}}^{(j, 0)}\right\|_{\boldsymbol{H}(\operatorname{curl}, \Omega)} & \leq C\left|D_{\rho_{n}}\right|^{1 / 2} .
\end{aligned}
$$

Furthermore, for any $\eta \in[1 / 5,1 / 2]$ there exists a constant $C_{\eta}$ such that

$$
\left\|\boldsymbol{w}_{\tilde{\varepsilon}_{\rho_{n}}}^{(j, 0)}-\boldsymbol{w}_{\tilde{\varepsilon}_{0}}^{(j)}\right\|_{L^{2}\left(\Omega, \mathbb{C}^{3}\right)} \leq C_{\eta}\left|D_{\rho_{n}}\right|^{1-\eta}
$$

Proof. This lemma can be shown using similar arguments as in the proof of Lemma 3.2.

To construct an explicit expression for $\operatorname{curl} \boldsymbol{w}_{\tilde{\varepsilon}_{0}}^{(j)}$ similar to (3.26) we observe that dive $\boldsymbol{e}_{j}=0$ in $\Omega$ and thus there exists a function $\boldsymbol{z}^{(j)} \in \boldsymbol{H}(\mathbf{c u r l}, \Omega)$ such that $\operatorname{curl} \boldsymbol{z}^{(j)}=\boldsymbol{e}_{j}$ (cf. [37], Thm. 3.38). The projection $P_{0} \boldsymbol{z}^{(j)}$ of this function onto $\boldsymbol{Y}_{0}^{\perp}$ satisfies (3.29), which means that $\boldsymbol{w}_{\tilde{\varepsilon}_{0}}^{(j)}=P_{0} \boldsymbol{z}^{(j)}$ and consequently

$$
\operatorname{curl} \boldsymbol{w}_{\tilde{\varepsilon}_{0}}^{(j)}=e_{j} .
$$


Lemma 3.8. For $1 \leq j \leq 3$ denote by $\boldsymbol{w}_{\tilde{\varepsilon}_{\rho_{n}}}^{(j)}, n \in \mathbb{N}$, the solution to (3.30). Then, there exists a subsequence, also denoted by $\left(D_{\rho_{n}}\right)_{n \in \mathbb{N}}$ and a matrix-valued function $\mathbb{M}^{\tilde{\varepsilon}}:=\left(M_{i j}^{\tilde{\varepsilon}}\right)_{1 \leq i, j \leq 3} \in \boldsymbol{L}^{2}\left(\Omega, \mathbb{C}^{3 \times 3} ; \mu\right)$, called electric polarization tensor, such that for $1 \leq i, j \leq 3$ and all $\phi \in C_{0}(\Omega, \mathbb{C})$,

$$
\int_{\Omega} \phi \frac{\tilde{\varepsilon}_{0}}{\tilde{\varepsilon}_{1}} \operatorname{curl} \boldsymbol{w}_{\tilde{\varepsilon}_{\rho_{n}}}^{(j)} \cdot \boldsymbol{e}_{i}\left|D_{\rho_{n}}\right|^{-1} \chi_{D_{\rho_{n}}} \mathrm{~d} \boldsymbol{x} \rightarrow \int_{\Omega} \phi M_{i j}^{\tilde{\varepsilon}} \mathrm{d} \mu \quad \text { as } n \rightarrow \infty .
$$

Proof. Proceeding as in [18], pp. 165-166, we use (3.34) and (3.36) to obtain

$$
\begin{aligned}
\left\|\left|D_{\rho_{n}}\right|^{-1} \chi_{D_{\rho_{n}}} \frac{\tilde{\varepsilon}_{0}}{\tilde{\varepsilon}_{1}} \operatorname{curl} \boldsymbol{w}_{\tilde{\varepsilon}_{\rho_{n}}}^{(j)}\right\|_{\boldsymbol{L}^{1}\left(\Omega, \mathbb{C}^{3}\right)} & \leq C\left|D_{\rho_{n}}\right|^{-1}\left(\left\|\operatorname{curl} \boldsymbol{w}_{\tilde{\varepsilon}_{\rho_{n}}}^{(j)}-\operatorname{curl} \boldsymbol{w}_{\tilde{\varepsilon}_{0}}^{(j)}\right\|_{\boldsymbol{L}^{1}\left(D_{\rho_{n}}, \mathbb{C}^{3}\right)}+\left\|\operatorname{curl} \boldsymbol{w}_{\tilde{\varepsilon}_{0}}^{(j)}\right\|_{\boldsymbol{L}^{1}\left(D_{\rho_{n}}, \mathbb{C}^{3}\right)}\right) \\
& \leq C\left|D_{\rho_{n}}\right|^{-1}\left(\|1\|_{L^{2}\left(D_{\rho_{n}}, \mathbb{C}\right)}\left\|\operatorname{curl} \boldsymbol{w}_{\tilde{\varepsilon}_{\rho_{n}}}^{(j)}-\operatorname{curl} \boldsymbol{w}_{\tilde{\varepsilon}_{0}}^{(j)}\right\|_{\boldsymbol{L}^{2}\left(\Omega, \mathbb{C}^{3}\right)}+\left|D_{\rho_{n}}\right|\right) \leq C .
\end{aligned}
$$

Therefore the Banach-Alaoglu Theorem yields a complex regular Borel measure $\mathcal{M}_{i j}^{\tilde{\varepsilon}}$ and a subsequence, again denoted by $\left(D_{\rho_{n}}\right)_{n \in \mathbb{N}}$, such that for all $\phi \in C_{0}(\Omega, \mathbb{C})$,

$$
\lim _{n \rightarrow \infty} \int_{\Omega} \phi \frac{\tilde{\varepsilon}_{0}}{\tilde{\varepsilon}_{1}} \operatorname{curl} \boldsymbol{w}_{\tilde{\varepsilon}_{\rho_{n}}}^{(j)} \cdot \boldsymbol{e}_{i}\left|D_{\rho_{n}}\right|^{-1} \chi_{D_{\rho_{n}}} \mathrm{~d} \boldsymbol{x}=\int_{\Omega} \phi \mathrm{d} \mathcal{M}_{i j}^{\tilde{\varepsilon}}
$$

Applying (3.34) and (3.36) once more we estimate

$$
\begin{aligned}
\left|\int_{\Omega} \phi \mathrm{d} \mathcal{M}_{i j}^{\tilde{\varepsilon}}\right| \leq & C\left(\liminf _{n \rightarrow \infty} \int_{\Omega}|\phi|\left|\left(\operatorname{curl} \boldsymbol{w}_{\tilde{\varepsilon}_{\rho_{n}}}^{(j)}-\operatorname{curl} \boldsymbol{w}_{\tilde{\varepsilon}_{0}}^{(j)}\right) \cdot \boldsymbol{e}_{i}\right|\left|D_{\rho_{n}}\right|^{-1} \chi_{D_{\rho_{n}}} \mathrm{~d} \boldsymbol{x}\right. \\
& \left.+\lim _{n \rightarrow \infty} \int_{\Omega}|\phi|\left|\operatorname{curl} \boldsymbol{w}_{\tilde{\varepsilon}_{0}}^{(j)} \cdot \boldsymbol{e}_{i}\right|\left|D_{\rho_{n}}\right|^{-1} \chi_{D_{\rho_{n}}} \mathrm{~d} \boldsymbol{x}\right) \\
\leq & C\left(\liminf _{n \rightarrow \infty}\left(\left|D_{\rho_{n}}\right|^{-1 / 2}\left\|\left(\operatorname{curl} \boldsymbol{w}_{\tilde{\varepsilon}_{\rho_{n}}}^{(j)}-\operatorname{curl}_{\tilde{\varepsilon}_{0}}^{(j)}\right) \cdot \boldsymbol{e}_{i}\right\|_{L^{2}(\Omega, \mathbb{C})}\left(\int_{\Omega}|\phi|^{2}\left|D_{\rho_{n}}\right|^{-1} \chi_{D_{\rho_{n}}} \mathrm{~d} \boldsymbol{x}\right)^{1 / 2}\right)\right. \\
& \left.\quad+\int_{\Omega}\left|\operatorname{curl} \boldsymbol{w}_{\tilde{\varepsilon}_{0}}^{(j)} \cdot \boldsymbol{e}_{i}\right||\phi| \mathrm{d} \mu\right) \\
\leq & C\|\phi\|_{L^{2}(\Omega, \mathbb{C} ; \mu)},
\end{aligned}
$$

where $L^{2}(\Omega, \mathbb{C} ; \mu)$ denotes the space of complex-valued square integrable functions on $\Omega$ with respect to the measure $\mu$. Therefore, $\phi \mapsto \int_{\Omega} \phi \mathrm{d} \mathcal{M}_{i j}^{\tilde{\varepsilon}}$ can be extended to a bounded linear functional on $L^{2}(\Omega, \mathbb{C} ; \mu)$ and from the Riesz Representation Theorem (cf., e.g., [36], Thm. 2.30) we obtain a unique $M_{i j}^{\tilde{\varepsilon}} \in L^{2}(\Omega, \mathbb{C} ; \mu)$ such that for all $\phi \in C_{0}(\Omega, \mathbb{C})$,

$$
\int_{\Omega} \phi \mathrm{d} \mathcal{M}_{i j}^{\tilde{\varepsilon}}=\int_{\Omega} \phi M_{i j}^{\tilde{\varepsilon}} \mathrm{d} \mu .
$$

Remark 3.9. For constant background permittivities $\tilde{\varepsilon}_{0}$ as considered in this work the definition of the electric polarization tensor $\mathbb{M} \tilde{\varepsilon}$ from (3.37) is equivalent to the definition of the magnetic polarization tensor $\mathbb{M}^{\mu}$ from (3.28) with $\mu_{\rho_{n}}$ replaced by $\tilde{\varepsilon}_{\rho_{n}}$ in the following sense: From (3.30) we find that

$$
\boldsymbol{F}:=\frac{1}{\tilde{\varepsilon}_{\rho_{n}}} \operatorname{curl} \boldsymbol{w}_{\tilde{\varepsilon}_{\rho_{n}}}^{(j)}-\frac{1}{\tilde{\varepsilon}_{0}} \boldsymbol{e}_{j} \in \boldsymbol{H}(\operatorname{curl}, \Omega)
$$

satisfies $\operatorname{curl} \boldsymbol{F}=0$ and $\boldsymbol{\nu} \times\left.\boldsymbol{F}\right|_{\partial \Omega}=0$. Therefore, $\boldsymbol{F}=\nabla p$ for some $p \in H_{0}^{1}(\Omega, \mathbb{C}):=\left\{f \in H^{1}(\Omega, \mathbb{C})|f|_{\partial \Omega}=0\right\}$ (cf. [37], Thm. 3.41), and accordingly

$$
\tilde{v}_{\tilde{\varepsilon}_{\rho_{n}}}^{(j)}:=\tilde{\varepsilon}_{0}\left(p+\frac{1}{\tilde{\varepsilon}_{0}} x_{j}\right)
$$


satisfies

$$
\operatorname{div}\left(\tilde{\varepsilon}_{\rho_{n}} \nabla \tilde{v}_{\tilde{\varepsilon}_{\rho_{n}}}^{(j)}\right)=0 \quad \text { in } \Omega, \quad \tilde{v}_{\tilde{\varepsilon}_{\rho_{n}}}^{(j)}=x_{j} \quad \text { on } \partial \Omega,
$$

which is of the same structure as (3.25) except for the boundary condition. Therewith,

$$
\frac{\tilde{\varepsilon}_{0}}{\tilde{\varepsilon}_{\rho_{n}}} \operatorname{curl} \boldsymbol{w}_{\tilde{\varepsilon}_{\rho_{n}}}^{(j)}=\nabla \tilde{\varepsilon}_{\tilde{\varepsilon}_{\rho_{n}}}^{(j)}
$$

and for any $\phi \in C_{0}(\Omega, \mathbb{C})(3.37)$ reduces to

$$
\int_{\Omega} \phi \frac{\partial \tilde{v}_{\tilde{\varepsilon}_{\rho_{n}}}^{(j)}}{\partial x_{i}}\left|D_{\rho_{n}}\right|^{-1} \chi_{D_{\rho_{n}}} \mathrm{~d} \boldsymbol{x} \rightarrow \int_{\Omega} \phi M_{i j}^{\tilde{\varepsilon}} \mathrm{d} \mu \quad \text { as } n \rightarrow \infty .
$$

It has been shown in [21], Lemma 1, that (3.38) (and therefore also (3.37)) yields the same polarization tensor as (3.28) with $v_{\mu_{\rho_{n}}}^{(j)}$ replaced by $v_{\tilde{\varepsilon}_{\rho_{n}}}^{(j)}$, where $v_{\tilde{\varepsilon}_{\rho_{n}}}^{(j)}$ denotes the solution to (3.25) with $\mu_{\rho_{n}}$ replaced by $\tilde{\varepsilon}_{\rho_{n}}$.

\section{The Perturbation Formula}

In this section we give the proof of Theorem 2.2. Throughout we assume that $\left(D_{\rho_{n}}\right)_{n \in \mathbb{N}}$ is a sequence of measurable subsets of $\Omega_{0} \subset \subset \Omega$ as in Section 2 such that $\left|D_{\rho_{n}}\right| \leq R$, where $R$ is the upper bound from Proposition 2.1, and (2.6), (3.28), and (3.37) are satisfied. We start by proving an integral representation for the trace of $\boldsymbol{H}_{\rho_{n}}-\boldsymbol{H}_{0}$ on $\partial \Omega$.

Lemma 4.1. Let $\boldsymbol{H}_{0}$ and $\boldsymbol{H}_{\rho_{n}}, n \in \mathbb{N}$, be the solutions of (2.3) and (2.4), respectively. Then, for any $\boldsymbol{y} \in \partial \Omega$,

$$
\begin{aligned}
\boldsymbol{\nu}(\boldsymbol{y}) & \times\left(\boldsymbol{H}_{\rho_{n}}-\boldsymbol{H}_{0}\right)(\boldsymbol{y})-2 \int_{\partial \Omega}\left(\boldsymbol{\nu}(\boldsymbol{y}) \times \operatorname{curl}_{x} \mathbb{G}(\cdot, \boldsymbol{y})\right)\left(\boldsymbol{\nu} \times\left(\boldsymbol{H}_{\rho_{n}}-\boldsymbol{H}_{0}\right)\right) \mathrm{d} s \\
& =2 \tilde{\varepsilon}_{0}\left(-\int_{\Omega}\left(\frac{1}{\tilde{\varepsilon}_{0}}-\frac{1}{\tilde{\varepsilon}_{\rho_{n}}}\right)\left(\boldsymbol{\nu}(\boldsymbol{y}) \times \operatorname{curl}_{x} \mathbb{G}(\cdot, \boldsymbol{y})\right) \operatorname{curl} \boldsymbol{H}_{\rho_{n}} \mathrm{~d} \boldsymbol{x}+\omega^{2} \int_{\Omega}\left(\mu_{0}-\mu_{\rho_{n}}\right)(\boldsymbol{\nu}(\boldsymbol{y}) \times \mathbb{G}(\cdot, \boldsymbol{y})) \boldsymbol{H}_{\rho_{n}} \mathrm{~d} \boldsymbol{x}\right) .
\end{aligned}
$$

Proof. First let $\boldsymbol{y} \in \Omega \backslash \overline{\Omega_{0}}$ be a point close to the boundary $\partial \Omega$ and choose $r>0$ small enough such that $B_{r}(\boldsymbol{y}) \subset \Omega \backslash \overline{\Omega_{0}}$. Using integration by parts we find that

$$
\begin{aligned}
0= & \int_{\Omega \backslash \overline{B_{r}(\boldsymbol{y})}}\left(\operatorname{curl}\left(\frac{1}{\tilde{\varepsilon}_{0}} \operatorname{curl}_{0}\right)-\omega^{2} \mu_{0} \boldsymbol{H}_{0}\right) \cdot \mathbb{G}(\cdot, \boldsymbol{y}) \mathrm{d} \boldsymbol{x} \\
= & \int_{\Omega \backslash \overline{B_{r}(\boldsymbol{y})}}\left(\frac{1}{\tilde{\varepsilon}_{0}} \operatorname{curl} \boldsymbol{H}_{0} \cdot \operatorname{curl}_{x} \mathbb{G}(\cdot, \boldsymbol{y})-\omega^{2} \mu_{0} \boldsymbol{H}_{0} \cdot \mathbb{G}(\cdot, \boldsymbol{y})\right) \mathrm{d} \boldsymbol{x} \\
& -\int_{\partial \Omega} \boldsymbol{g} \cdot \mathbb{G}(\cdot, \boldsymbol{y}) \mathrm{d} s+\int_{\partial B_{r}(\boldsymbol{y})}\left(\frac{1}{\tilde{\varepsilon}_{0}} \operatorname{curl} \boldsymbol{H}_{0} \times \boldsymbol{\nu}\right) \cdot \mathbb{G}(\cdot, \boldsymbol{y}) \mathrm{d} s
\end{aligned}
$$

and similarly

$$
\begin{aligned}
0= & \int_{\Omega \backslash \overline{B_{r}(\boldsymbol{y})}}\left(\frac{1}{\tilde{\varepsilon}_{\rho_{n}}} \operatorname{curl}_{\boldsymbol{H}_{\rho_{n}}} \cdot \operatorname{curl}_{x} \mathbb{G}(\cdot, \boldsymbol{y})-\omega^{2} \mu_{\rho_{n}} \boldsymbol{H}_{\rho_{n}} \cdot \mathbb{G}(\cdot, \boldsymbol{y})\right) \mathrm{d} \boldsymbol{x} \\
& -\int_{\partial \Omega} \boldsymbol{g} \cdot \mathbb{G}(\cdot, \boldsymbol{y}) \mathrm{d} s+\int_{\partial B_{r}(\boldsymbol{y})}\left(\frac{1}{\tilde{\varepsilon}_{\rho_{n}}} \operatorname{curl} \boldsymbol{H}_{\rho_{n}} \times \boldsymbol{\nu}\right) \cdot \mathbb{G}(\cdot, \boldsymbol{y}) \mathrm{d} s .
\end{aligned}
$$


Subtracting these formulas we obtain

$$
\begin{aligned}
& \int_{\Omega \backslash \overline{B_{r}(\boldsymbol{y})}}\left(\frac{1}{\tilde{\varepsilon}_{0}} \operatorname{curl}\left(\boldsymbol{H}_{\rho_{n}}-\boldsymbol{H}_{0}\right) \cdot \operatorname{curl}_{x} \mathbb{G}(\cdot, \boldsymbol{y})-\omega^{2} \mu_{0}\left(\boldsymbol{H}_{\rho_{n}}-\boldsymbol{H}_{0}\right) \cdot \mathbb{G}(\cdot, \boldsymbol{y})\right) \mathrm{d} \boldsymbol{x} \\
& =\int_{\Omega \backslash \overline{B_{r}(\boldsymbol{y})}}\left(\left(\frac{1}{\tilde{\varepsilon}_{0}}-\frac{1}{\tilde{\varepsilon}_{\rho_{n}}}\right) \operatorname{curl} \boldsymbol{H}_{\rho_{n}} \cdot \operatorname{curl}_{x} \mathbb{G}(\cdot, \boldsymbol{y})-\omega^{2}\left(\mu_{0}-\mu_{\rho_{n}}\right) \boldsymbol{H}_{\rho_{n}} \cdot \mathbb{G}(\cdot, \boldsymbol{y})\right) \mathrm{d} \boldsymbol{x} \\
& +\int_{\partial B_{r}(\boldsymbol{y})} \frac{1}{\tilde{\varepsilon}_{0}}\left(\operatorname{curl} \boldsymbol{H}_{0} \times \boldsymbol{\nu}\right) \cdot \mathbb{G}(\cdot, \boldsymbol{y}) \mathrm{d} s-\int_{\partial B_{r}(\boldsymbol{y})} \frac{1}{\tilde{\varepsilon}_{\rho_{n}}}\left(\operatorname{curl} \boldsymbol{H}_{\rho_{n}} \times \boldsymbol{\nu}\right) \cdot \mathbb{G}(\cdot, \boldsymbol{y}) \mathrm{d} s .
\end{aligned}
$$

Integrating by parts once more the left hand side of (4.2) can be rewritten as

$$
\begin{aligned}
& \int_{\Omega \backslash \overline{B_{r}(\boldsymbol{y})}}\left(\frac{1}{\tilde{\varepsilon}_{0}} \operatorname{curl}\left(\boldsymbol{H}_{\rho_{n}}-\boldsymbol{H}_{0}\right) \cdot \operatorname{curl}_{x} \mathbb{G}(\cdot, \boldsymbol{y})-\omega^{2} \mu_{0}\left(\boldsymbol{H}_{\rho_{n}}-\boldsymbol{H}_{0}\right) \cdot \mathbb{G}(\cdot, \boldsymbol{y})\right) \mathrm{d} \boldsymbol{x} \\
& =-\int_{\partial \Omega} \frac{1}{\tilde{\varepsilon}_{0}}\left(\left(\boldsymbol{H}_{\rho_{n}}-\boldsymbol{H}_{0}\right) \times \boldsymbol{\nu}\right) \cdot \operatorname{curl}_{x} \mathbb{G}(\cdot, \boldsymbol{y}) \mathrm{d} s+\int_{\partial B_{r}(\boldsymbol{y})} \frac{1}{\tilde{\varepsilon}_{0}}\left(\left(\boldsymbol{H}_{\rho_{n}}-\boldsymbol{H}_{0}\right) \times \boldsymbol{\nu}\right) \cdot \operatorname{curl}_{x} \mathbb{G}(\cdot, \boldsymbol{y}) \mathrm{d} s .
\end{aligned}
$$

Combining (4.2) and (4.3), observing that $\tilde{\varepsilon}_{0}$ and $\tilde{\varepsilon}_{\rho_{n}}$ as well as $\mu_{0}$ and $\mu_{\rho_{n}}$ coincide on $\overline{B_{r}(\boldsymbol{y})}$, and applying the Stratton-Chu Formula (see, e.g., [37], Thm. 9.2) we find that

$$
\begin{aligned}
\int_{\Omega}\left(\left(\frac{1}{\tilde{\varepsilon}_{0}}-\frac{1}{\tilde{\varepsilon}_{\rho_{n}}}\right)\right. & \left.\operatorname{curl} \boldsymbol{H}_{\rho_{n}} \cdot \operatorname{curl}_{x} \mathbb{G}(\cdot, \boldsymbol{y})-\omega^{2}\left(\mu_{0}-\mu_{\rho_{n}}\right) \boldsymbol{H}_{\rho_{n}} \cdot \mathbb{G}(\cdot, \boldsymbol{y})\right) \mathrm{d} \boldsymbol{x} \\
= & \left.\frac{1}{\tilde{\varepsilon}_{0}} \int_{\partial B_{r}(\boldsymbol{y})}\left(\left(\operatorname{curl}\left(\boldsymbol{H}_{\rho_{n}}-\boldsymbol{H}_{0}\right)\right) \times \boldsymbol{\nu}\right) \cdot \mathbb{G}(\cdot, \boldsymbol{y})+\left(\left(\boldsymbol{H}_{\rho_{n}}-\boldsymbol{H}_{0}\right) \times \boldsymbol{\nu}\right) \cdot \operatorname{curl}_{x} \mathbb{G}(\cdot, \boldsymbol{y})\right) \mathrm{d} s \\
& -\frac{1}{\tilde{\varepsilon}_{0}} \int_{\partial \Omega}\left(\left(\boldsymbol{H}_{\rho_{n}}-\boldsymbol{H}_{0}\right) \times \boldsymbol{\nu}\right) \cdot \operatorname{curl}_{x} \mathbb{G}(\cdot, \boldsymbol{y}) \mathrm{d} s \\
= & \frac{1}{\tilde{\varepsilon}_{0}}\left(\boldsymbol{H}_{\rho_{n}}-\boldsymbol{H}_{0}\right)^{\top}(\boldsymbol{y})-\frac{1}{\tilde{\varepsilon}_{0}} \int_{\partial \Omega}\left(\left(\boldsymbol{H}_{\rho_{n}}-\boldsymbol{H}_{0}\right) \times \boldsymbol{\nu}\right) \cdot \operatorname{curl}_{x} \mathbb{G}(\cdot, \boldsymbol{y}) \mathrm{d} s
\end{aligned}
$$

Now letting $\boldsymbol{y} \rightarrow \partial \Omega$, using the trace formula for curls of vector potentials (see Colton and Kress [24], Thm. 2.26), and recalling that $\mathbb{G}(\cdot, \boldsymbol{y})$ is symmetric while $\operatorname{curl}_{x} \mathbb{G}(\cdot, \boldsymbol{y})$ is skew symmetric yields (4.1).

Next, inspired by the proof of [18], Lemma 2, we use an integration by parts technique to decompose the influence of the boundary data and of the geometry of the scatterer on the magnetic field $\boldsymbol{H}_{\rho_{n}}$ as $n \rightarrow \infty$.

Lemma 4.2. Let $\boldsymbol{H}_{0}$ and $\boldsymbol{H}_{\rho_{n}}$ be the solutions of (2.3) and (2.4), respectively. Then, for $1 \leq j \leq 3$ and all $\phi \in C_{0}(\Omega, \mathbb{C})$,

$$
\begin{aligned}
& \lim _{n \rightarrow \infty}\left(\int_{\Omega} \phi\left(\frac{1}{\tilde{\varepsilon}_{0}}-\frac{1}{\tilde{\varepsilon}_{1}}\right) \operatorname{curl} \boldsymbol{H}_{\rho_{n}} \cdot\right.\left.\boldsymbol{e}_{j}\left|D_{\rho_{n}}\right|^{-1} \chi_{D_{\rho_{n}}} \mathrm{~d} \boldsymbol{x}-\omega^{2} \int_{\Omega} \phi\left(\mu_{0}-\mu_{1}\right) \boldsymbol{H}_{\rho_{n}} \cdot \boldsymbol{e}_{j}\left|D_{\rho_{n}}\right|^{-1} \chi_{D_{\rho_{n}}} \mathrm{~d} \boldsymbol{x}\right) \\
&=\int_{\Omega} \phi \frac{\tilde{\varepsilon}_{1}}{\tilde{\varepsilon}_{0}}\left(\frac{1}{\tilde{\varepsilon}_{0}}-\frac{1}{\tilde{\varepsilon}_{1}}\right) \operatorname{curl} \boldsymbol{H}_{0} \cdot \mathbb{M}_{-j}^{\tilde{\varepsilon}} \mathrm{d} \mu-\omega^{2} \int_{\Omega} \phi\left(\mu_{0}-\mu_{1}\right) \boldsymbol{H}_{0} \cdot \mathbb{M}_{-j}^{\mu} \mathrm{d} \mu
\end{aligned}
$$

where $\mathbb{M}_{-j}^{\tilde{\varepsilon}}$ and $\mathbb{M}_{-j}^{\mu}$ denote the $j$ th column of the electric and magnetic polarization tensor, respectively. 
Proof. Subtracting the weak formulations (2.3) and (2.4) with test function $\boldsymbol{v}=\overline{\boldsymbol{w}_{\tilde{\varepsilon}_{0}}^{(j)} \phi}$ and applying (3.4) and (3.7) gives

$$
\begin{aligned}
& \int_{\Omega}\left(\frac{1}{\tilde{\varepsilon}_{0}}-\frac{1}{\tilde{\varepsilon}_{\rho_{n}}}\right) \operatorname{curl} \boldsymbol{H}_{\rho_{n}} \cdot \operatorname{curl}\left(\boldsymbol{w}_{\tilde{\varepsilon}_{0}}^{(j)} \phi\right) \mathrm{d} \boldsymbol{x}-\omega^{2} \int_{\Omega}\left(\mu_{0}-\mu_{\rho_{n}}\right) \boldsymbol{H}_{\rho_{n}} \cdot \boldsymbol{w}_{\tilde{\varepsilon}_{0}}^{(j)} \phi \mathrm{d} \boldsymbol{x} \\
& =\int_{\Omega} \frac{1}{\tilde{\varepsilon}_{0}} \operatorname{curl}\left(\boldsymbol{H}_{\rho_{n}}-\boldsymbol{H}_{0}\right) \cdot \operatorname{curl}\left(\boldsymbol{w}_{\tilde{\varepsilon}_{0}}^{(j)} \phi\right) \mathrm{d} \boldsymbol{x}-\omega^{2} \int_{\Omega} \mu_{0}\left(\boldsymbol{H}_{\rho_{n}}-\boldsymbol{H}_{0}\right) \cdot \boldsymbol{w}_{\tilde{\varepsilon}_{0}}^{(j)} \phi \mathrm{d} \boldsymbol{x} \\
& =\int_{\Omega} \frac{1}{\tilde{\varepsilon}_{0}} \operatorname{curl}\left(\boldsymbol{h}_{\rho_{n}}^{(0)}-\boldsymbol{h}_{0}\right) \cdot \operatorname{curl}\left(\boldsymbol{w}_{\tilde{\varepsilon}_{0}}^{(j)} \phi\right) \mathrm{d} \boldsymbol{x}-\omega^{2} \int_{\Omega} \mu_{0}\left(\boldsymbol{H}_{\rho_{n}}-\boldsymbol{H}_{0}\right) \cdot \boldsymbol{w}_{\tilde{\varepsilon}_{0}}^{(j)} \phi \mathrm{d} \boldsymbol{x}
\end{aligned}
$$

and similarly for $\boldsymbol{v}=\overline{\boldsymbol{w}_{\tilde{\varepsilon}_{\rho_{n}}}^{(j, 0)} \phi}$,

$$
\begin{aligned}
& \int_{\Omega}\left(\frac{1}{\tilde{\varepsilon}_{0}}-\frac{1}{\tilde{\varepsilon}_{\rho_{n}}}\right) \operatorname{curl} \boldsymbol{H}_{0} \cdot \operatorname{curl}\left(\boldsymbol{w}_{\tilde{\varepsilon}_{\rho_{n}}}^{(j, 0)} \phi\right) \mathrm{d} \boldsymbol{x}-\omega^{2} \int_{\Omega}\left(\mu_{0}-\mu_{\rho_{n}}\right) \boldsymbol{H}_{0} \cdot \boldsymbol{w}_{\tilde{\varepsilon}_{\rho_{n}}}^{(j, 0)} \phi \mathrm{d} \boldsymbol{x} \\
& =\int_{\Omega} \frac{1}{\tilde{\varepsilon}_{\rho_{n}}} \operatorname{curl}\left(\boldsymbol{H}_{\rho_{n}}-\boldsymbol{H}_{0}\right) \cdot \operatorname{curl}\left(\boldsymbol{w}_{\tilde{\varepsilon}_{\rho_{n}}}^{(j, 0)} \phi\right) \mathrm{d} \boldsymbol{x}-\omega^{2} \int_{\Omega} \mu_{\rho_{n}}\left(\boldsymbol{H}_{\rho_{n}}-\boldsymbol{H}_{0}\right) \cdot \boldsymbol{w}_{\tilde{\varepsilon}_{\rho_{n}}}^{(j, 0)} \phi \mathrm{d} \boldsymbol{x} \\
& =\int_{\Omega} \frac{1}{\tilde{\varepsilon}_{\rho_{n}}} \operatorname{curl}\left(\boldsymbol{h}_{\rho_{n}}^{(0)}-\boldsymbol{h}_{0}\right) \cdot \operatorname{curl}\left(\boldsymbol{w}_{\tilde{\varepsilon}_{\rho_{n}}}^{(j, 0)} \phi\right) \mathrm{d} \boldsymbol{x}-\omega^{2} \int_{\Omega} \mu_{\rho_{n}}\left(\boldsymbol{H}_{\rho_{n}}-\boldsymbol{H}_{0}\right) \cdot \boldsymbol{w}_{\tilde{\varepsilon}_{\rho_{n}}}^{(j, 0)} \phi \mathrm{d} \boldsymbol{x}
\end{aligned}
$$

On the other hand, using the test function $\boldsymbol{v}=\overline{\nabla\left(\boldsymbol{v}_{\mu_{0}}^{(j)} \phi\right)}$ we obtain that

$$
\begin{aligned}
\int_{\Omega}\left(\mu_{0}-\mu_{\rho_{n}}\right) \boldsymbol{H}_{\rho_{n}} \cdot \nabla\left(v_{\mu_{0}}^{(j)} \phi\right) \mathrm{d} \boldsymbol{x} & =\int_{\Omega} \mu_{0}\left(\boldsymbol{H}_{\rho_{n}}-\boldsymbol{H}_{0}\right) \cdot \nabla\left(v_{\mu_{0}}^{(j)} \phi\right) \mathrm{d} \boldsymbol{x} \\
& =\int_{\Omega} \mu_{0} \nabla\left(q_{\rho_{n}}^{(0)}-q_{0}\right) \cdot \nabla\left(v_{\mu_{0}}^{(j)} \phi\right) \mathrm{d} \boldsymbol{x}
\end{aligned}
$$

and similarly, applying (3.4) and (3.7) we find for $\boldsymbol{v}=\overline{\nabla\left(\boldsymbol{v}_{\mu_{\rho_{n}}}^{(j)} \phi\right)}$ that

$$
\begin{aligned}
\int_{\Omega}\left(\mu_{0}-\mu_{\rho_{n}}\right) \boldsymbol{H}_{0} \cdot \nabla\left(v_{\mu_{\rho_{n}}}^{(j)} \phi\right) \mathrm{d} \boldsymbol{x}= & \int_{\Omega} \mu_{\rho_{n}}\left(\boldsymbol{H}_{\rho_{n}}-\boldsymbol{H}_{0}\right) \cdot \nabla\left(v_{\mu_{\rho_{n}}}^{(j)} \phi\right) \mathrm{d} \boldsymbol{x} \\
= & \int_{\Omega} \mu_{\rho_{n}} \nabla\left(q_{\rho_{n}}^{(0)}-q_{0}\right) \cdot \nabla\left(v_{\mu_{\rho_{n}}}^{(j)} \phi\right) \mathrm{d} \boldsymbol{x}+\int_{\Omega}\left(\mu_{\rho_{n}}-\mu_{0}\right)\left(\boldsymbol{h}_{\rho_{n}}^{(0)}-\boldsymbol{h}_{0}\right) \cdot \nabla\left(v_{\mu_{0}}^{(j)} \phi\right) \mathrm{d} \boldsymbol{x} \\
& +\int_{\Omega} \mu_{\rho_{n}}\left(\boldsymbol{h}_{\rho_{n}}^{(0)}-\boldsymbol{h}_{0}\right) \cdot \nabla\left(\left(v_{\mu_{\rho_{n}}}^{(j)}-v_{\mu_{0}}^{(j)}\right) \phi\right) \mathrm{d} \boldsymbol{x} \\
= & \int_{\Omega} \mu_{\rho_{n}} \nabla\left(q_{\rho_{n}}^{(0)}-q_{0}\right) \cdot \nabla\left(v_{\mu_{\rho_{n}}}^{(j)} \phi\right) \mathrm{d} \boldsymbol{x}+o\left(\left|D_{\rho_{n}}\right|\right) .
\end{aligned}
$$

Here we used orthogonality, (3.13), (3.27a), and the smoothness of $v_{\mu_{0}}^{(j)}$ in $\Omega_{0}$. 
Recalling (3.12) and (3.35) we can calculate

$$
\begin{aligned}
& \int_{\Omega} \frac{1}{\tilde{\varepsilon}_{\rho_{n}}} \operatorname{curl}\left(\boldsymbol{h}_{\rho_{n}}^{(0)}-\boldsymbol{h}_{0}\right) \cdot \operatorname{curl}\left(\boldsymbol{w}_{\tilde{\varepsilon}_{\rho_{n}}}^{(j, 0)} \phi\right) \mathrm{d} \boldsymbol{x}=\int_{\Omega} \frac{1}{\tilde{\varepsilon}_{\rho_{n}}} \operatorname{curl}\left(\boldsymbol{h}_{\rho_{n}}^{(0)}-\boldsymbol{h}_{0}\right) \cdot \operatorname{curl}\left(\boldsymbol{w}_{\tilde{\varepsilon}_{\rho_{n}}}^{(j)}\right) \phi \mathrm{d} \boldsymbol{x} \\
&+\int_{\Omega} \frac{1}{\tilde{\varepsilon}_{\rho_{n}}} \operatorname{curl}\left(\boldsymbol{h}_{\rho_{n}}^{(0)}-\boldsymbol{h}_{0}\right) \cdot\left(\nabla \phi \times \boldsymbol{w}_{\tilde{\varepsilon}_{0}}^{(j)}\right) \mathrm{d} \boldsymbol{x}+o\left(\left|D_{\rho_{n}}\right|\right) \\
&=\int_{\Omega} \frac{1}{\tilde{\varepsilon}_{\rho_{n}}} \operatorname{curl}\left(\boldsymbol{h}_{\rho_{n}}^{(0)}-\boldsymbol{h}_{0}\right) \cdot \operatorname{curl}\left(\boldsymbol{w}_{\tilde{\varepsilon}_{\rho_{n}}}^{(j)}\right) \phi \mathrm{d} \boldsymbol{x}+\int_{\Omega} \frac{1}{\tilde{\varepsilon}_{0}} \operatorname{curl}\left(\boldsymbol{h}_{\rho_{n}}^{(0)}-\boldsymbol{h}_{0}\right) \cdot\left(\nabla \phi \times \boldsymbol{w}_{\tilde{\varepsilon}_{0}}^{(j)}\right) \mathrm{d} \boldsymbol{x} \\
&+\int_{\Omega}\left(\frac{1}{\tilde{\varepsilon}_{\rho_{n}}}-\frac{1}{\tilde{\varepsilon}_{0}}\right) \operatorname{curl}\left(\boldsymbol{h}_{\rho_{n}}^{(0)}-\boldsymbol{h}_{0}\right) \cdot\left(\nabla \phi \times \boldsymbol{w}_{\tilde{\varepsilon}_{0}}^{(j)}\right) \mathrm{d} \boldsymbol{x}+o\left(\left|D_{\rho_{n}}\right|\right) .
\end{aligned}
$$

Integrating by parts and using (3.30), (3.13), and (3.34) yields

$$
\begin{aligned}
\int_{\Omega} & \frac{1}{\tilde{\varepsilon}_{\rho_{n}}} \operatorname{curl}\left(\boldsymbol{h}_{\rho_{n}}^{(0)}-\boldsymbol{h}_{0}\right) \cdot \operatorname{curl}\left(\boldsymbol{w}_{\tilde{\varepsilon}_{\rho_{n}}}^{(j, 0)} \phi\right) \mathrm{d} \boldsymbol{x} \\
= & \int_{\Omega}\left(\boldsymbol{h}_{\rho_{n}}^{(0)}-\boldsymbol{h}_{0}\right) \cdot \operatorname{curl}\left(\frac{1}{\tilde{\varepsilon}_{\rho_{n}}} \operatorname{curl}\left(\boldsymbol{w}_{\tilde{\varepsilon}_{\rho_{n}}}^{(j)}\right) \phi\right) \mathrm{d} \boldsymbol{x}+\int_{\partial \Omega}\left(\boldsymbol{h}_{\rho_{n}}^{(0)}-\boldsymbol{h}_{0}\right) \cdot\left(\frac{1}{\tilde{\varepsilon}_{\rho_{n}}}\left(\operatorname{curl} \boldsymbol{w}_{\tilde{\varepsilon}_{\rho_{n}}}^{(j)}\right) \times \boldsymbol{\nu} \phi\right) \mathrm{d} s \\
& +\int_{\Omega} \frac{1}{\tilde{\varepsilon}_{0}} \operatorname{curl}\left(\boldsymbol{h}_{\rho_{n}}^{(0)}-\boldsymbol{h}_{0}\right) \cdot\left(\nabla \phi \times \boldsymbol{w}_{\tilde{\varepsilon}_{0}}^{(j)}\right) \mathrm{d} \boldsymbol{x}+\int_{\Omega}\left(\frac{1}{\tilde{\varepsilon}_{\rho_{n}}}-\frac{1}{\tilde{\varepsilon}_{0}}\right) \operatorname{curl}\left(\boldsymbol{h}_{\rho_{n}}^{(0)}-\boldsymbol{h}_{0}\right) \cdot\left(\nabla \phi \times \boldsymbol{w}_{\tilde{\varepsilon}_{0}}^{(j)}\right) \mathrm{d} \boldsymbol{x}+o\left(\left|D_{\rho_{n}}\right|\right) \\
= & \int_{\Omega} \frac{1}{\tilde{\varepsilon}_{\rho_{n}}}\left(\boldsymbol{h}_{\rho_{n}}^{(0)}-\boldsymbol{h}_{0}\right) \cdot\left(\nabla \phi \times \operatorname{curl} \boldsymbol{w}_{\tilde{\varepsilon}_{\rho_{n}}}^{(j)}\right) \mathrm{d} \boldsymbol{x}+\int_{\partial \Omega}\left(\boldsymbol{h}_{\rho_{n}}^{(0)}-\boldsymbol{h}_{0}\right) \cdot\left(\frac{1}{\tilde{\varepsilon}_{0}} \boldsymbol{e}_{j} \times \boldsymbol{\nu}\right) \phi \mathrm{d} s \\
& +\int_{\Omega} \frac{1}{\tilde{\varepsilon}_{0}} \operatorname{curl}\left(\boldsymbol{h}_{\rho_{n}}^{(0)}-\boldsymbol{h}_{0}\right) \cdot\left(\nabla \phi \times \boldsymbol{w}_{\tilde{\varepsilon}_{0}}^{(j)}\right) \mathrm{d} \boldsymbol{x}+\int_{\Omega}\left(\frac{1}{\tilde{\varepsilon}_{\rho_{n}}}-\frac{1}{\tilde{\varepsilon}_{0}}\right) \operatorname{curl}\left(\boldsymbol{h}_{\rho_{n}}^{(0)}-\boldsymbol{h}_{0}\right) \cdot\left(\nabla \phi \times \boldsymbol{w}_{\tilde{\varepsilon}_{0}}^{(j)}\right) \mathrm{d} \boldsymbol{x}+o\left(\left|D_{\rho_{n}}\right|\right) \\
= & \int_{\Omega} \frac{1}{\tilde{\varepsilon}_{\rho_{n}}}\left(\boldsymbol{h}_{\rho_{n}}^{(0)}-\boldsymbol{h}_{0}\right) \cdot\left(\nabla \phi \times \operatorname{curl} \boldsymbol{w}_{\tilde{\varepsilon}_{0}}^{(j)}\right) \mathrm{d} \boldsymbol{x}+\int_{\partial \Omega}\left(\boldsymbol{h}_{\rho_{n}}^{(0)}-\boldsymbol{h}_{0}\right) \cdot\left(\frac{1}{\tilde{\varepsilon}_{0}} \boldsymbol{e}_{j} \times \boldsymbol{\nu}\right) \phi \mathrm{d} s \\
& +\int_{\Omega} \frac{1}{\tilde{\varepsilon}_{0}} \operatorname{curl}\left(\boldsymbol{h}_{\rho_{n}}^{(0)}-\boldsymbol{h}_{0}\right) \cdot\left(\nabla \phi \times \boldsymbol{w}_{\tilde{\varepsilon}_{0}}^{(j)}\right) \mathrm{d} \boldsymbol{x}+\int_{\Omega}\left(\frac{1}{\tilde{\varepsilon}_{\rho_{n}}}-\frac{1}{\tilde{\varepsilon}_{0}}\right) \operatorname{curl}\left(\boldsymbol{h}_{\rho_{n}}^{(0)}-\boldsymbol{h}_{0}\right) \cdot\left(\nabla \phi \times \boldsymbol{w}_{\tilde{\varepsilon}_{0}}^{(j)}\right) \mathrm{d} \boldsymbol{x}+o\left(\left|D_{\rho_{n}}\right|\right) .
\end{aligned}
$$

Analogously,

$$
\begin{aligned}
\int_{\Omega} \frac{1}{\tilde{\varepsilon}_{0}} \operatorname{curl}\left(\boldsymbol{h}_{\rho_{n}}^{(0)}-\boldsymbol{h}_{0}\right) \cdot \operatorname{curl}\left(\boldsymbol{w}_{\tilde{\varepsilon}_{0}}^{(j)} \phi\right) \mathrm{d} \boldsymbol{x} & =\int_{\Omega} \frac{1}{\tilde{\varepsilon}_{0}}\left(\boldsymbol{h}_{\rho_{n}}^{(0)}-\boldsymbol{h}_{0}\right) \cdot\left(\nabla \phi \times \operatorname{curl} \boldsymbol{w}_{\tilde{\varepsilon}_{0}}^{(j)}\right) \mathrm{d} \boldsymbol{x} \\
& +\int_{\partial \Omega}\left(\boldsymbol{h}_{\rho_{n}}^{(0)}-\boldsymbol{h}_{0}\right) \cdot\left(\frac{1}{\tilde{\varepsilon}_{0}} \boldsymbol{e}_{j} \times \boldsymbol{\nu}\right) \phi \mathrm{d} s+\int_{\Omega} \frac{1}{\tilde{\varepsilon}_{0}} \operatorname{curl}\left(\boldsymbol{h}_{\rho_{n}}^{(0)}-\boldsymbol{h}_{0}\right) \cdot\left(\nabla \phi \times \boldsymbol{w}_{\tilde{\varepsilon}_{0}}^{(j)}\right) \mathrm{d} \boldsymbol{x} .
\end{aligned}
$$

Combining these two equations we find that

$$
\begin{aligned}
\int_{\Omega} \frac{1}{\tilde{\varepsilon}_{\rho_{n}}} \operatorname{curl}\left(\boldsymbol{h}_{\rho_{n}}^{(0)}-\boldsymbol{h}_{0}\right) \cdot \operatorname{curl}\left(\boldsymbol{w}_{\tilde{\varepsilon}_{\rho_{n}}}^{(j, 0)} \phi\right) \mathrm{d} \boldsymbol{x} & =\int_{\Omega} \frac{1}{\tilde{\varepsilon}_{0}} \operatorname{curl}\left(\boldsymbol{h}_{\rho_{n}}^{(0)}-\boldsymbol{h}_{0}\right) \cdot \operatorname{curl}\left(\boldsymbol{w}_{\tilde{\varepsilon}_{0}}^{(j)} \phi\right) \mathrm{d} \boldsymbol{x} \\
& +\int_{\Omega}\left(\frac{1}{\tilde{\varepsilon}_{\rho_{n}}}-\frac{1}{\tilde{\varepsilon}_{0}}\right) \operatorname{curl}\left(\boldsymbol{h}_{\rho_{n}}^{(0)}-\boldsymbol{h}_{0}\right) \cdot\left(\nabla \phi \times \boldsymbol{w}_{\tilde{\varepsilon}_{0}}^{(j)}\right) \mathrm{d} \boldsymbol{x}+o\left(\left|D_{\rho_{n}}\right|\right) .
\end{aligned}
$$


Similar arguments give

$$
\begin{aligned}
\int_{\Omega} \mu_{\rho_{n}} \nabla\left(q_{\rho_{n}}^{(0)}-q_{0}\right) \cdot \nabla\left(v_{\mu_{n}}^{(j)} \phi\right) \mathrm{d} \boldsymbol{x}=\int_{\Omega} \mu_{0} \nabla\left(q_{\rho_{n}}^{(0)}-\right. & \left.q_{0}\right) \cdot \nabla\left(v_{\mu_{0}}^{(j)} \phi\right) \mathrm{d} \boldsymbol{x} \\
& \quad+\int_{\Omega}\left(\mu_{\rho_{n}}-\mu_{0}\right) \nabla\left(q_{\rho_{n}}^{(0)}-q_{0}\right) \cdot(\nabla \phi) v_{\mu_{0}}^{(j)} \mathrm{d} \boldsymbol{x}+o\left(\left|D_{\rho_{n}}\right|\right)
\end{aligned}
$$

(see the proof of [18], Lem. 2 for details).

Now substituting (4.9) into (4.6) and applying (4.5) we find that

$$
\begin{aligned}
& \int_{\Omega}\left(\frac{1}{\tilde{\varepsilon}_{0}}-\frac{1}{\tilde{\varepsilon}_{\rho_{n}}}\right) \operatorname{curl} \boldsymbol{H}_{0} \cdot \operatorname{curl}\left(\boldsymbol{w}_{\tilde{\varepsilon}_{\rho_{n}}}^{(j, 0)} \phi\right) \mathrm{d} \boldsymbol{x}-\omega^{2} \int_{\Omega}\left(\mu_{0}-\mu_{\rho_{n}}\right) \boldsymbol{H}_{0} \cdot \boldsymbol{w}_{\tilde{\varepsilon}_{\rho_{n}}}^{(j, 0)} \phi \mathrm{d} \boldsymbol{x} \\
& +\omega^{2} \int_{\Omega} \mu_{\rho_{n}}\left(\boldsymbol{H}_{\rho_{n}}-\boldsymbol{H}_{0}\right) \cdot \boldsymbol{w}_{\tilde{\varepsilon}_{\rho_{n}}}^{(j, 0)} \phi \mathrm{d} \boldsymbol{x} \\
& =\int_{\Omega} \frac{1}{\tilde{\varepsilon}_{0}} \operatorname{curl}\left(\boldsymbol{h}_{\rho_{n}}^{(0)}-\boldsymbol{h}_{0}\right) \cdot \operatorname{curl}\left(\boldsymbol{w}_{\tilde{\varepsilon}_{0}}^{(j)} \phi\right) \mathrm{d} \boldsymbol{x}+\int_{\Omega}\left(\frac{1}{\tilde{\varepsilon}_{\rho_{n}}}-\frac{1}{\tilde{\varepsilon}_{0}}\right) \operatorname{curl}\left(\boldsymbol{h}_{\rho_{n}}^{(0)}-\boldsymbol{h}_{0}\right) \cdot\left(\nabla \phi \times \boldsymbol{w}_{\tilde{\varepsilon}_{0}}^{(j)}\right) \mathrm{d} \boldsymbol{x}+o\left(\left|D_{\rho_{n}}\right|\right) \\
& =\int_{\Omega}\left(\frac{1}{\tilde{\varepsilon}_{0}}-\frac{1}{\tilde{\varepsilon}_{\rho_{n}}}\right) \operatorname{curl} \boldsymbol{H}_{\rho_{n}} \cdot \operatorname{curl}\left(\boldsymbol{w}_{\tilde{\varepsilon}_{0}}^{(j)} \phi\right) \mathrm{d} \boldsymbol{x}-\omega^{2} \int_{\Omega}\left(\mu_{0}-\mu_{\rho_{n}}\right) \boldsymbol{H}_{\rho_{n}} \cdot \boldsymbol{w}_{\tilde{\varepsilon}_{0}}^{(j)} \phi \mathrm{d} \boldsymbol{x} \\
& +\omega^{2} \int_{\Omega} \mu_{0}\left(\boldsymbol{H}_{\rho_{n}}-\boldsymbol{H}_{0}\right) \cdot \boldsymbol{w}_{\tilde{\varepsilon}_{0}}^{(j)} \phi \mathrm{d} \boldsymbol{x}-\int_{\Omega}\left(\frac{1}{\tilde{\varepsilon}_{0}}-\frac{1}{\tilde{\varepsilon}_{\rho_{n}}}\right) \operatorname{curl} \boldsymbol{H}_{\rho_{n}} \cdot\left(\nabla \phi \times \boldsymbol{w}_{\tilde{\varepsilon}_{0}}^{(j)}\right) \mathrm{d} \boldsymbol{x} \\
& +\int_{\Omega}\left(\frac{1}{\tilde{\varepsilon}_{0}}-\frac{1}{\tilde{\varepsilon}_{\rho_{n}}}\right) \operatorname{curl} \boldsymbol{H}_{0} \cdot\left(\nabla \phi \times \boldsymbol{w}_{\tilde{\varepsilon}_{\rho_{n}}}^{(j, 0)}\right) \mathrm{d} \boldsymbol{x}+o\left(\left|D_{\rho_{n}}\right|\right) .
\end{aligned}
$$

In the last step we used (3.35) to obtain

$$
\left|\int_{\Omega}\left(\frac{1}{\tilde{\varepsilon}_{0}}-\frac{1}{\tilde{\varepsilon}_{\rho_{n}}}\right) \operatorname{curl} \boldsymbol{H}_{0} \cdot\left(\nabla \phi \times\left(\boldsymbol{w}_{\tilde{\varepsilon}_{\rho_{n}}}^{(j, 0)}-\boldsymbol{w}_{\tilde{\varepsilon}_{0}}^{(j)}\right)\right) \mathrm{d} \boldsymbol{x}\right| \leq C_{\eta}\left|D_{\rho_{n}}\right|^{3 / 2-\eta}
$$

Using (3.10), (3.12), and (3.35) we can estimate

$$
\begin{aligned}
& \mid-\omega^{2} \int_{\Omega}\left(\mu_{0}-\mu_{\rho_{n}}\right) \boldsymbol{H}_{0} \cdot \boldsymbol{w}_{\tilde{\varepsilon}_{\rho_{n}}}^{(j, 0)} \phi \mathrm{d} \boldsymbol{x}+\omega^{2} \int_{\Omega} \mu_{\rho_{n}}\left(\boldsymbol{H}_{\rho_{n}}-\boldsymbol{H}_{0}\right) \cdot \boldsymbol{w}_{\tilde{\varepsilon}_{\rho_{n}}}^{(j, 0)} \phi \mathrm{d} \boldsymbol{x} \\
&+\omega^{2} \int_{\Omega}\left(\mu_{0}-\mu_{\rho_{n}}\right) \boldsymbol{H}_{\rho_{n}} \cdot \boldsymbol{w}_{\tilde{\varepsilon}_{0}}^{(j)} \phi \mathrm{d} \boldsymbol{x}-\omega^{2} \int_{\Omega} \mu_{0}\left(\boldsymbol{H}_{\rho_{n}}-\boldsymbol{H}_{0}\right) \cdot \boldsymbol{w}_{\tilde{\varepsilon}_{0}}^{(j)} \phi \mathrm{d} \boldsymbol{x} \mid \\
&=\mid-\omega^{2} \int_{\Omega}\left(\mu_{0}-\mu_{\rho_{n}}\right) \boldsymbol{H}_{0} \cdot\left(\boldsymbol{w}_{\tilde{\varepsilon}_{\rho_{n}}}^{(j, 0)}-\boldsymbol{w}_{\tilde{\varepsilon}_{0}}^{(j)}\right) \phi \mathrm{d} \boldsymbol{x}-\omega^{2} \int_{\Omega}\left(\mu_{0}-\mu_{\rho_{n}}\right) \boldsymbol{H}_{0} \cdot \boldsymbol{w}_{\tilde{\varepsilon}_{0}}^{(j)} \phi \mathrm{d} \boldsymbol{x} \\
&+\omega^{2} \int_{\Omega} \mu_{\rho_{n}}\left(\boldsymbol{H}_{\rho_{n}}-\boldsymbol{H}_{0}\right) \cdot\left(\boldsymbol{w}_{\tilde{\varepsilon}_{\rho_{n}}}^{(j, 0)}-\boldsymbol{w}_{\tilde{\varepsilon}_{0}}^{(j)}\right) \phi \mathrm{d} \boldsymbol{x}+\omega^{2} \int_{\Omega} \mu_{\rho_{n}}\left(\boldsymbol{H}_{\rho_{n}}-\boldsymbol{H}_{0}\right) \cdot \boldsymbol{w}_{\tilde{\varepsilon}_{0}}^{(j)} \phi \mathrm{d} \boldsymbol{x} \\
&=\left|\omega^{2} \int_{\Omega}\left(\mu_{0}-\mu_{\rho_{n}}\right) \boldsymbol{H}_{\rho_{n}} \cdot \boldsymbol{w}_{\tilde{\varepsilon}_{0}}^{(j)} \phi \mathrm{d} \boldsymbol{x}-\omega^{2} \int_{\Omega} \mu_{0}\left(\boldsymbol{H}_{\rho_{n}}-\boldsymbol{H}_{0}\right) \cdot \boldsymbol{w}_{\tilde{\varepsilon}_{0}}^{(j)} \phi \mathrm{d} \boldsymbol{x}\right| \\
&= o\left(\left|D_{\rho_{n}}\right|\right) .
\end{aligned}
$$


Thus, after rearrangement and rescaling (4.11) reduces to

$$
\begin{aligned}
& \int_{\Omega}\left(\frac{1}{\tilde{\varepsilon}_{0}}-\frac{1}{\tilde{\varepsilon}_{1}}\right) \operatorname{curl} \boldsymbol{H}_{0} \cdot \operatorname{curl}\left(\boldsymbol{w}_{\tilde{\varepsilon}_{\rho_{n}}}^{(j)}\right) \phi\left|D_{\rho_{n}}\right|^{-1} \chi_{D_{\rho_{n}}} \mathrm{~d} \boldsymbol{x} \\
&=\int_{\Omega}\left(\frac{1}{\tilde{\varepsilon}_{0}}-\frac{1}{\tilde{\varepsilon}_{1}}\right) \operatorname{curl}_{\rho_{n}} \cdot \operatorname{curl}\left(\boldsymbol{w}_{\tilde{\varepsilon}_{0}}^{(j)}\right) \phi\left|D_{\rho_{n}}\right|^{-1} \chi_{D_{\rho_{n}}} \mathrm{~d} \boldsymbol{x}+o(1) .
\end{aligned}
$$

Similarly, substituting (4.10) into (4.8) and applying (4.7) yields

$$
\begin{aligned}
\int_{\Omega}\left(\mu_{0}-\mu_{\rho_{n}}\right) & \boldsymbol{H}_{0} \cdot \nabla\left(v_{\mu_{\rho_{n}}}^{(j)} \phi\right) \mathrm{d} \boldsymbol{x} \\
& =\int_{\Omega} \mu_{0} \nabla\left(q_{\rho_{n}}^{(0)}-q_{0}\right) \cdot \nabla\left(v_{\mu_{0}}^{(j)} \phi\right) \mathrm{d} \boldsymbol{x}+\int_{\Omega}\left(\mu_{\rho_{n}}-\mu_{0}\right) \nabla\left(q_{\rho_{n}}^{(0)}-q_{0}\right) \cdot(\nabla \phi) v_{\mu_{0}}^{(j)} \mathrm{d} \boldsymbol{x}+o\left(\left|D_{\rho_{n}}\right|\right) \\
& =\int_{\Omega}\left(\mu_{0}-\mu_{\rho_{n}}\right) \boldsymbol{H}_{\rho_{n}} \cdot \nabla\left(v_{\mu_{0}}^{(j)} \phi\right) \mathrm{d} \boldsymbol{x}-\int_{\Omega}\left(\mu_{0}-\mu_{\rho_{n}}\right) \nabla\left(q_{\rho_{n}}^{(0)}-q_{0}\right) \cdot(\nabla \phi) v_{\mu_{0}}^{(j)} \mathrm{d} \boldsymbol{x}+o\left(\left|D_{\rho_{n}}\right|\right) .
\end{aligned}
$$

From (3.13) we observe that

$$
\int_{\Omega}\left(\mu_{0}-\mu_{\rho_{n}}\right)\left(\boldsymbol{h}_{\rho_{n}}^{(0)}-\boldsymbol{h}_{0}\right) \cdot(\nabla \phi) v_{\mu_{0}}^{(j)} \mathrm{d} \boldsymbol{x}=o\left(\left|D_{\rho_{n}}\right|\right)
$$

and use $(3.27 \mathrm{~b})$ to obtain

$$
\begin{aligned}
\int_{\Omega}\left(\mu_{0}-\mu_{\rho_{n}}\right) \boldsymbol{H}_{0} \cdot \nabla\left(v_{\mu_{\rho_{n}}}^{(j)} \phi\right) \mathrm{d} \boldsymbol{x}= & \int_{\Omega}\left(\mu_{0}-\mu_{\rho_{n}}\right) \boldsymbol{H}_{\rho_{n}} \cdot \nabla\left(v_{\mu_{0}}^{(j)} \phi\right) \mathrm{d} \boldsymbol{x}-\int_{\Omega}\left(\mu_{0}-\mu_{\rho_{n}}\right) \boldsymbol{H}_{\rho_{n}} \cdot(\nabla \phi) v_{\mu_{0}}^{(j)} \mathrm{d} \boldsymbol{x} \\
& +\int_{\Omega}\left(\mu_{0}-\mu_{\rho_{n}}\right) \boldsymbol{H}_{0} \cdot(\nabla \phi) v_{\mu_{\rho_{n}}}^{(j)} \mathrm{d} \boldsymbol{x}+o\left(\left|D_{\rho_{n}}\right|\right) .
\end{aligned}
$$

Therefore, rearrangement and rescaling gives

$$
\begin{array}{rl}
-\omega^{2} \int_{\Omega}\left(\mu_{0}-\mu_{1}\right) \boldsymbol{H}_{0} \cdot\left(\nabla v_{\mu_{\rho_{n}}}^{(j)}\right) \phi \chi_{D_{\rho_{n}}}\left|D_{\rho_{n}}\right|^{-1} & \mathrm{~d} \boldsymbol{x} \\
& =-\omega^{2} \int_{\Omega}\left(\mu_{0}-\mu_{1}\right) \boldsymbol{H}_{\rho_{n}} \cdot\left(\nabla v_{\mu_{0}}^{(j)}\right) \phi \chi_{D_{\rho_{n}}}\left|D_{\rho_{n}}\right|^{-1} \mathrm{~d} \boldsymbol{x}+o(1) .
\end{array}
$$

Letting $n \rightarrow \infty$ in (4.12)-(4.4) follows by applying (3.28), (3.37) and the formulas (3.26) and (3.36).

Proof of Theorem 2.2. Let $\left(D_{\rho_{n}}\right)_{n \in \mathbb{N}}$ be a sequence as in Theorem 2.2. Then we can extract a subsequence, also denoted by $\left(D_{\rho_{n}}\right)_{n \in \mathbb{N}}$, for which (2.6), (3.28), and (3.37) hold. Rescaling (4.1) we find that for $\boldsymbol{y} \in \partial \Omega$ and any $n \in \mathbb{N}$,

$$
\begin{aligned}
\boldsymbol{\nu}(\boldsymbol{y}) \times\left(\boldsymbol{H}_{\rho_{n}}-\boldsymbol{H}_{0}\right)(\boldsymbol{y})-2 \int_{\partial \Omega}\left(\boldsymbol{\nu}(\boldsymbol{y}) \times \operatorname{curl}_{x} \mathbb{G}(\cdot, \boldsymbol{y})\right)\left(\boldsymbol{\nu} \times\left(\boldsymbol{H}_{\rho_{n}}-\boldsymbol{H}_{0}\right)\right) \mathrm{d} s & \\
=\left|D_{\rho_{n}}\right| 2 \tilde{\varepsilon}_{0}\left(-\int_{\Omega}\left(\frac{1}{\tilde{\varepsilon}_{0}}-\frac{1}{\tilde{\varepsilon}_{1}}\right)(\boldsymbol{\nu}(\boldsymbol{y})\right. & \left.\times \operatorname{curl}_{x} \mathbb{G}(\cdot, \boldsymbol{y})\right) \operatorname{curl} \boldsymbol{H}_{\rho_{n}}\left|D_{\rho_{n}}\right|^{-1} \chi_{D_{\rho_{n}}} \mathrm{~d} \boldsymbol{x} \\
& \left.\quad+\omega^{2} \int_{\Omega}\left(\mu_{0}-\mu_{1}\right)(\boldsymbol{\nu}(\boldsymbol{y}) \times \mathbb{G}(\cdot, \boldsymbol{y})) \boldsymbol{H}_{\rho_{n}}\left|D_{\rho_{n}}\right|^{-1} \chi_{D_{\rho_{n}}} \mathrm{~d} \boldsymbol{x}\right) .
\end{aligned}
$$


Since $\boldsymbol{\nu}(\boldsymbol{y}) \times \mathbb{G}(\cdot, \boldsymbol{y})$ and $\boldsymbol{\nu}(\boldsymbol{y}) \times \operatorname{curl} \mathbb{G}(\cdot, \boldsymbol{y})$ are smooth in $\Omega_{0}$ for any $\boldsymbol{y} \in \partial \Omega$ and their restrictions to $\Omega_{0}$ can be extended to continuous functions in $C_{0}(\Omega)$, we can apply (4.4) to obtain $(2.7)$.

\section{EXAMPLES AND APPLICATIONS}

Given an arbitrary sequence $\left(D_{\rho_{n}}\right)_{n \in \mathbb{N}}$ of low volume scatterers as introduced in Section 2, Theorem 2.2 guarantees the existence of a limiting measure $\mu$ and of polarization tensors $\mathbb{M} \tilde{\varepsilon}$ and $\mathbb{M}^{\mu}$ corresponding to an appropriate subsequence such that the representation formula (2.7) holds, but neither the limiting measure nor the polarization tensors are independent of the particular subsequence in general. However, as we discussed in Remarks 3.6 and 3.9, $\mu$ as well as $\mathbb{M}^{\mu}$ and $\mathbb{M}^{\tilde{\varepsilon}}$ are equivalent to the corresponding limiting measure and polarization tensor appearing in the representation formula for voltage perturbations caused by low volume conductivity inhomogeneities from [18]. These have been very well studied in the past twenty years and explicit characterizations of the limiting measure and of the polarization tensor are known for several practically interesting examples. In the following we consider three of them, show how these earlier results apply to our setting, and discuss how the corresponding asymptotic representation formulas have been used or can be used to solve inverse scattering problems. Throughout let $\left(\rho_{n}\right)_{n \in \mathbb{N}}$ be a sequence of sufficiently small positive real numbers converging to zero.

Example 5.1 (Point Scatterers). In our first example we consider a collection of $m$ diametrically small scatterers $D_{\rho_{n}}:=\bigcup_{l=1}^{m}\left(\boldsymbol{z}_{l}+\rho_{n} B_{l}\right)$ that are well separated from each other and from the boundary of $\Omega$. Here $B_{1}, \ldots, B_{m} \subset$ $\mathbb{R}^{3}$ are bounded smooth domains containing the origin that determine the relative shape of the scatterers. The location of the scatterers is given by the points $\boldsymbol{z}_{1}, \ldots, \boldsymbol{z}_{m} \in \Omega$, and their diameter is specified by $\rho_{n}$.

As mentioned before, this particular example has already been studied for time-harmonic Maxwell's equations in [9]. It follows straightforwardly from (2.6) that in this case the limiting measure $\mu$ is given by $\mu=\frac{1}{\sum_{l=1}^{m}\left|B_{l}\right|} \sum_{l=1}^{m}\left|B_{l}\right| \delta_{\boldsymbol{z}_{l}}$, where $\delta_{\boldsymbol{z}_{l}}$ denotes the Dirac measure with support in $\boldsymbol{z}_{l}$, and that it is not necessary to consider a particular subsequence. The asymptotic representation formula (2.7) reduces for any $\boldsymbol{y} \in \partial \Omega$ to

$$
\begin{aligned}
& \boldsymbol{\nu}(\boldsymbol{y}) \times\left(\boldsymbol{H}_{\rho_{n}}-\right.\left.\boldsymbol{H}_{0}\right)(\boldsymbol{y})-2 \int_{\partial \Omega}\left(\boldsymbol{\nu}(\boldsymbol{y}) \times \operatorname{curl}_{x} \mathbb{G}(\cdot, \boldsymbol{y})\right)\left(\boldsymbol{\nu} \times\left(\boldsymbol{H}_{\rho_{n}}-\boldsymbol{H}_{0}\right)\right) \mathrm{d} s \\
&=\rho_{n}{ }^{3} 2 \tilde{\varepsilon}_{0} \sum_{l=1}^{m}\left|B_{l}\right|\left(-\left(\frac{\tilde{\varepsilon}_{1}}{\tilde{\varepsilon}_{0}}\left(\frac{1}{\tilde{\varepsilon}_{0}}-\frac{1}{\tilde{\varepsilon}_{1}}\right)\right)\left(\boldsymbol{z}_{l}\right)\left(\boldsymbol{\nu}(\boldsymbol{y}) \times \operatorname{curl}_{x} \mathbb{G}\left(\boldsymbol{z}_{l}, \boldsymbol{y}\right)\right) \mathbb{M} \tilde{\varepsilon}_{l}^{\tilde{\varepsilon}} \operatorname{curl} \boldsymbol{H}_{0}\left(\boldsymbol{z}_{l}\right)\right. \\
&\left.\quad+\omega^{2}\left(\mu_{0}-\mu_{1}\right)\left(\boldsymbol{z}_{l}\right)\left(\boldsymbol{\nu}(\boldsymbol{y}) \times \mathbb{G}\left(\boldsymbol{z}_{l}, \boldsymbol{y}\right)\right) \mathbb{M}_{l}^{\mu} \boldsymbol{H}_{0}\left(\boldsymbol{z}_{l}\right)\right)+o\left(\rho_{n}{ }^{3}\right),
\end{aligned}
$$

where $\mathbb{M}_{l}^{\mu}$ and $\mathbb{M}_{l}^{\tilde{\varepsilon}}, 1 \leq l \leq m$, are the polarization tensors associated with the sequence of single scatterers $\left(\boldsymbol{z}_{l}+\rho_{n} B_{l}\right)_{n \in \mathbb{N}}$ defined similar to (3.28) and (3.37), respectively. The sequences (3.28) and (3.37) have been shown to converge without extracting a particular subsequence for this example (see, e.g., [9,22]), i.e., the asymptotic formula (5.1) holds without extracting a subsequence. Explicit formulas for $\mathbb{M}_{l}^{\mu}$ and $\mathbb{M}_{l}^{\tilde{\varepsilon}}, 1 \leq l \leq m$, are available if $B_{l}$ is a ball or an ellipsoid (cf., e.g., [5]).

Extensions of this formula to unbounded domains have been considered in [11,28], and reconstruction methods for the corresponding inverse scattering problem in unbounded domains have been studied in [2,28,29,31-33]. Assuming that the diameter of the scatterers described by $\rho_{n}$ is small, these methods neglect the $o\left(\rho_{n}{ }^{3}\right)$ term in (5.1) and describe the measured electromagnetic fields by the leading order term in this formula. Then, observing that the unique continuation of the leading order term to the interior of $\Omega$ has singularities at the locations $\boldsymbol{z}_{1}, \ldots, \boldsymbol{z}_{m}$ of the small scatterers, the inverse problem to reconstruct the positions of the scatterers reduces to the inverse problem to recover the singularities of this unique continuation from its boundary data. A popular method to do so is the so-called MUSIC algorithm (see, e.g., Cheney [23]). 
Example 5.2 (thin tubes). Let $K_{1}, \ldots, K_{m}$ be a collection of $m$ simple smooth curves in $\Omega$ that are well separated from each other and from the boundary of $\Omega$. We consider a family of scatterers $D_{\rho_{n}}:=\bigcup_{l=1}^{m} D_{\rho_{n}, l}$ such that $D_{\rho_{n}, l}:=\left\{\boldsymbol{x} \in \Omega \mid \operatorname{dist}\left(\boldsymbol{x}, K_{l}\right)<\rho_{n} / 2\right\}$ is a tubular neighborhood around $K_{l}$ and $\rho_{n}$ determines its diameter. These thin tubular scatterers can be used to model the practically interesting problem of electromagnetic scattering from thin wires or pipes.

The limit measure $\mu$ for this example has been derived in $[13,30]$. Convergence takes place without extracting a particular subsequence and $\mu=\frac{1}{\sum_{l=1}^{m}\left|K_{l}\right|} \sum_{l=1}^{m} \delta_{K_{l}}$, where $\left|K_{l}\right|$ denotes the length of the curve $K_{l}$ and the measure $\delta_{K_{l}}$ is defined by

$$
\int_{\Omega} \phi(\boldsymbol{x}) \mathrm{d} \delta_{K_{l}}(\boldsymbol{x})=\int_{K_{l}} \phi(\boldsymbol{x}) \mathrm{d} \lambda(\boldsymbol{x}) \quad \text { for all } \phi \in C_{0}(\Omega, \mathbb{C})
$$

Here the right hand side has to be understood in the sense of curve integrals. So, for any $\boldsymbol{y} \in \partial \Omega$, formula (2.7) reduces to

$$
\begin{array}{r}
\boldsymbol{\nu}(\boldsymbol{y}) \times\left(\boldsymbol{H}_{\rho_{n}}-\boldsymbol{H}_{0}\right)(\boldsymbol{y})-2 \int_{\partial \Omega}\left(\boldsymbol{\nu}(\boldsymbol{y}) \times \operatorname{curl}_{x} \mathbb{G}(\cdot, \boldsymbol{y})\right)\left(\boldsymbol{\nu} \times\left(\boldsymbol{H}_{\rho_{n}}-\boldsymbol{H}_{0}\right)\right) \mathrm{d} s \\
=\rho_{n}{ }^{2} 2 \tilde{\varepsilon}_{0} \sum_{l=1}^{m} \int_{K_{l}}\left(-\frac{\tilde{\varepsilon}_{1}}{\tilde{\varepsilon}_{0}}\left(\frac{1}{\tilde{\varepsilon}_{0}}-\frac{1}{\tilde{\varepsilon}_{1}}\right)\left(\boldsymbol{\nu}(\boldsymbol{y}) \times \operatorname{curl}_{x} \mathbb{G}(\cdot, \boldsymbol{y})\right) \mathbb{M}_{l}^{\tilde{\varepsilon}} \mathbf{c u r l} \boldsymbol{H}_{0}\right. \\
\left.+\omega^{2}\left(\mu_{0}-\mu_{1}\right)(\boldsymbol{\nu}(\boldsymbol{y}) \times \mathbb{G}(\cdot, \boldsymbol{y})) \mathbb{M}_{l}^{\mu} \boldsymbol{H}_{0}\right) \mathrm{d} \lambda+o\left(\rho_{n}{ }^{2}\right),
\end{array}
$$

where $\mathbb{M}_{l}^{\mu}$ and $\mathbb{M}_{l}^{\tilde{\varepsilon}}, 1 \leq l \leq m$, are the polarization tensors associated with the sequence of single scatterers $\left(D_{\rho_{n}, l}\right)_{n \in \mathbb{N}}$ defined similar to $(3.28)$ and (3.37), respectively.

For the special case when $K_{1}, \ldots, K_{m}$ are straight line segments and the medium is non-conducting, the polarization tensors $\mathbb{M}_{l}^{\mu}$ and $\mathbb{M}_{l}^{\tilde{\varepsilon}}$ have been characterized in [13]. For any $\boldsymbol{x} \in K_{l}$, the matrix $\mathbb{M}_{l}^{\gamma}(\boldsymbol{x}), \gamma \in\{\mu, \tilde{\varepsilon}\}$, is symmetric and positive definite with eigenvalue 1 of multiplicity one and eigenvalue $\left(\left(2 \gamma_{0}\right) /\left(\gamma_{0}+\gamma_{1}\right)\right)(\boldsymbol{x})$ of multiplicity two. The eigenvector corresponding to the eigenvalue 1 is tangential to $K_{l}$ in $\boldsymbol{x}$. In this special case the polarization tensors are independent of the particular subsequence, i.e., (5.2) holds without taking a subsequence.

First reconstruction methods for the corresponding inverse problem have recently been studied for the electrostatic case. In [13] a collection of appropriately chosen input data has been used to recover the position and orientation of a single thin straight cylindrical conductivity inhomogeneity directly from the formula for the leading order term in the asymptotic expansion of the perturbation of electrostatic potentials similar to (5.2). On the other hand in [30] the position of multiple arbitrarily curved thin tubular conductivity inhomogeneities has been detected by reconstructing the singularities of the unique continuation of leading order term of the asymptotic formula to the interior of the domain. A variant of the factorization method (see, e.g., Kirsch and Grinberg [35]) has been used to find these singularities. Based on (5.2) a similar method can be developed for the corresponding fully three-dimensional inverse electromagnetic scattering problem.

Example 5.3 (thin sheets). Let $S_{1}, \ldots, S_{m}$ be a collection of $m$ simple hypersurfaces in $\Omega$ that are well separated from each other and from the boundary of $\Omega$. We consider a family of scatterers $D_{\rho_{n}}:=\bigcup_{l=1}^{m} D_{\rho_{n}, l}$ such that $D_{\rho_{n}, l}:=\left\{\boldsymbol{x} \in \Omega \mid \operatorname{dist}\left(\boldsymbol{x}, S_{l}\right)<\rho_{n} / 2\right\}$ is a neighborhood of the hypersurface $S_{l}$ and $\rho_{n}$ determines its thickness. These thin sheet-like scatterers can be used as a model for thin penetrable cracks in non-destructive testing. 
The limiting measure $\mu$ for this example has been derived in $[15,16,21]$, where a corresponding electrostatic problem has been considered (see also [14]). The sequence of measures (2.5) converges without extracting a subsequence to $\mu=\frac{1}{\sum_{l=1}^{m}\left|S_{l}\right|} \sum_{l=1}^{m} \delta_{S_{l}}$, where $\left|S_{l}\right|$ denotes the area of the surface $S_{l}$ and the measure $\delta_{S_{l}}$ is defined by

$$
\int_{\Omega} \phi(\boldsymbol{x}) \mathrm{d} \delta_{S_{l}}(\boldsymbol{x})=\int_{S_{l}} \phi(\boldsymbol{x}) \mathrm{d} s(\boldsymbol{x}) \quad \text { for all } \phi \in C_{0}(\Omega, \mathbb{C}) .
$$

Here the right hand side has to be understood in the sense of surface integrals. So, formula (2.7) reduces for any $\boldsymbol{y} \in \partial \Omega$ to

$$
\begin{array}{r}
\boldsymbol{\nu}(\boldsymbol{y}) \times\left(\boldsymbol{H}_{\rho_{n}}-\boldsymbol{H}_{0}\right)(\boldsymbol{y})-2 \int_{\partial \Omega}\left(\boldsymbol{\nu}(\boldsymbol{y}) \times \operatorname{curl}_{x} \mathbb{G}(\cdot, \boldsymbol{y})\right)\left(\boldsymbol{\nu} \times\left(\boldsymbol{H}_{\rho_{n}}-\boldsymbol{H}_{0}\right)\right) \mathrm{d} s \\
=\rho_{n} 2 \tilde{\varepsilon}_{0} \sum_{l=1}^{m} \int_{S_{l}}\left(-\frac{\tilde{\varepsilon}_{1}}{\tilde{\varepsilon}_{0}}\left(\frac{1}{\tilde{\varepsilon}_{0}}-\frac{1}{\tilde{\varepsilon}_{1}}\right)\left(\boldsymbol{\nu}(\boldsymbol{y}) \times \operatorname{curl}_{x} \mathbb{G}(\cdot, \boldsymbol{y})\right) \mathbb{M}_{l}^{\tilde{\varepsilon}} \mathbf{c u r l} \boldsymbol{H}_{0}\right. \\
\left.+\omega^{2}\left(\mu_{0}-\mu_{1}\right)(\boldsymbol{\nu}(\boldsymbol{y}) \times \mathbb{G}(\cdot, \boldsymbol{y})) \mathbb{M}_{l}^{\mu} \boldsymbol{H}_{0}\right) \mathrm{d} s+o\left(\rho_{n}\right),
\end{array}
$$

where $\mathbb{M}_{l}^{\mu}$ and $\mathbb{M}_{l}^{\tilde{\varepsilon}}, 1 \leq l \leq m$, are the polarization tensors associated with the sequence of single scatterers $\left(D_{\rho_{n}, l}\right)_{n \in \mathbb{N}}$ defined similar to (3.28) and (3.37), respectively.

For real-valued coefficients these tensors have been characterized in [15,16,21]. In this case, for any $\boldsymbol{x} \in S_{l}$ the matrix $\mathbb{M}_{l}^{\gamma}(\boldsymbol{x}), \gamma \in\{\mu, \tilde{\varepsilon}\}$, is symmetric and positive definite with a two-dimensional eigenspace corresponding to the eigenvalue 1 that is tangential to $S_{l}$ in $\boldsymbol{x}$ and a one-dimensional eigenspace corresponding to the eigenvalue $\left(\gamma_{0} / \gamma_{1}\right)(\boldsymbol{x})$. In particular, the sequences (3.28) and (3.37) have been shown to converge without extracting a subsequence for this example and therefore the asymptotic formula (5.1) holds without extracting a subsequence.

Although no reconstruction methods for this inverse scattering problem have been considered so far, (5.3) suggests a starting point to do so.

\section{Concluding REMARKS}

Throughout we restricted our attention to homogeneous background media with constant parameters $\varepsilon_{0}$, $\sigma_{0}$, and $\mu_{0}$. To extend our analysis to inhomogeneous background media with smoothly varying parameters $\varepsilon_{0}, \sigma_{0}, \mu_{0} \in C^{\infty}(\bar{\Omega}, \mathbb{R})$ that are uniformly bounded from above and below (i.e., $0<c_{\mu_{0}} \leq \mu_{0} \leq C_{\mu_{0}}<\infty$, $0<c_{\varepsilon_{0}} \leq \varepsilon_{0} \leq C_{\varepsilon_{0}}<\infty$, and $0 \leq \sigma_{0} \leq C_{\sigma_{0}}<\infty$ for some constants $c_{\mu_{0}}, C_{\mu_{0}}, c_{\varepsilon_{0}}, C_{\varepsilon_{0}}$, and $\left.C_{\sigma_{0}}\right)$ only Lemma 4.1 and its proof need to be modified. The estimates in Section 3 and their proofs remain true without changes, and also the proof of Lemma 4.2 carries over straightforwardly. However, if $\tilde{\varepsilon}_{0}$ is non-constant, the arguments used in Remark 3.9 to relate the definition of the electric polarization tensor $\mathbb{M}^{\tilde{\varepsilon}}$ to the definition of the magnetic polarization tensor $\mathbb{M}^{\mu}$ and the polarization tensor appearing in the electrostatic case fail.

A closely related open problem is to prove an asymptotic formula for perturbations in the eigenfrequencies of the Maxwell system (2.1) caused by general low volume scatterers as considered in this work. Corresponding results for diametrically small scatterers as in Example 5.1 have been established by Ammari and Volkov [10].

Acknowledgements. The author is pleased to thank Prof. Peter Monk and Prof. Michael Vogelius for their support and for interesting discussions in the course of this project.

\section{REFERENCES}

[1] R.A. Adams, Sobolev Spaces, Pure Appl. Math. 65. Academic Press, New York (1975).

[2] H. Ammari, E. Iakovleva, D. Lesselier and G. Perrusson, MUSIC-type electromagnetic imaging of a collection of small threedimensional bounded inclusions. SIAM J. Sci. Comput. 29 (2007) 674-709.

[3] H. Ammari and H. Kang, High-order terms in the asymptotic expansions of the steady-state voltage potentials in the presence of conductivity inhomogeneities of small diameter. SIAM J. Math. Anal. 34 (2003) 1152-1166. 
[4] H. Ammari and H. Kang, Boundary layer techniques for solving the Helmholtz equation in the presence of small inhomogeneities. J. Math. Anal. Appl. 296 (2004) 190-208.

[5] H. Ammari and H. Kang, Polarization and Moment Tensors with Applications to Inverse Problems and Effective Medium Theory, Appl. Math. Sci. 162. Springer-Verlag, Berlin (2007).

[6] H. Ammari and A. Khelifi, Electromagnetic scattering by small dielectric inhomogeneities. J. Math. Pures Appl. 82 (2003) 749-842.

[7] H. Ammari, S. Moskow and M.S. Vogelius, Boundary integral formulae for the reconstruction of electric and electromagnetic inhomogeneities of small volume. ESAIM: COCV 9 (2003) 49-66.

[8] H. Ammari and J.K. Seo, An accurate formula for the reconstruction of conductivity inhomogeneities. Adv. Appl. Math. 30 (2003) 679-705.

[9] H. Ammari, M.S. Vogelius and D. Volkov, Asymptotic formulas for perturbations in the electromagnetic fields due to the presence of inhomogeneities of small diameter II. the full Maxwell equations. J. Math. Pures Appl. 80 (2001) 769-814.

[10] H. Ammari and D. Volkov, Asymptotic formulas for perturbations in the eigenfrequencies of the full maxwell equations due to the presence of imperfections of small diameter. Asympt. Anal. 30 (2002) 331-350.

[11] H. Ammari and D. Volkov, The leading order term in the asymptotic expansion of the scattering amplitude of a collection of finite number of dielectric inhomogeneities of small diameter. Int. J. Multiscale Comput. Engrg. 3 (2005) 149-160.

[12] D.N. Arnold, R.S. Falk and R. Winther, Multigrid in H(div) and H(curl). Numer. Math. 85 (2000) 197-217.

[13] E. Beretta, Y. Capdeboscq, F. de Gournay and E. Francini, Thin cylindrical conductivity inclusions in a 3-dimensional domain: a polarization tensor and unique determination from boundary data. Inverse Problems 25 (2009) 065004.

[14] E. Beretta and E. Francini, Asymptotic formulas for perturbations in the electromagnetic fields due to the presence of thin inhomogeneities Contemp. Math. 333, edited by G. Uhlmann and G. Alessandrini, Amer. Math. Soc., Providence (2003).

[15] E. Beretta, E. Francini and M.S. Vogelius, Asymptotic formulas for steady state voltage potentials in the presence of thin inhomogeneities. a rigorous error analysis. J. Math. Pures Appl. 82 (2003) 1277-1301.

[16] E. Beretta, A. Mukherjee and M.S. Vogelius, Asymptotic formulas for steady state voltage potentials in the presence of conductivity imperfections of small area. Z. Angew. Math. Phys. 52 (2001) 543-572.

[17] M. Brühl, M. Hanke and M.S. Vogelius, A direct impedance tomography algorithm for locating small inhomogeneities. Numer. Math. 93 (2003) 635-654.

[18] Y. Capdeboscq and M.S. Vogelius, A general representation formula for boundary voltage perturbations caused by internal conductivity inhomogeneities of low volume fraction. Math. Model. Numer. Anal. 37 (2003) 159-173.

[19] Y. Capdeboscq and M.S. Vogelius, Optimal asymptotic estimates for the volume of internal inhomogeneities in terms of multiple boundary measurements. Math. Model. Numer. Anal. 37 (2003) 227-240.

[20] Y. Capdeboscq and M.S. Vogelius, A review of some recent work on impedance imaging for inhomogeneities of low volume fraction. Contemp. Math. 362, edited by C. Conca, R. Manasevich, G. Uhlmann and M.S. Vogelius, Amer. Math. Soc., Providence (2004).

[21] Y. Capdeboscq and M.S. Vogelius, Pointwise polarization tensor bounds, and applications to voltage perturbations caused by thin inhomogeneities. Asymptot. Anal. 50 (2006) 175-204.

[22] D. Cedio-Fengya, S. Moskow and M.S. Vogelius, Identification of conductivity imperfections of small diameter by boundary measurements. Continuous dependence and computational reconstruction. Inverse Problems 14 (1998) 553-595.

[23] M. Cheney, The linear sampling method and the MUSIC algorithm. Inverse Problems 17 (2001) 591-595.

[24] D. Colton and R. Kress, Integral Equation Methods in Scattering Theory. John Wiley \& Sons, New York (1983).

[25] R. Dautray and J.-L. Lions, Mathematical Analysis and Numerical Methods for Science and Technology. Spectral Theory and Applications 3. Springer-Verlag, Berlin (1990).

[26] A. Friedman and M.S. Vogelius, Identification of small inhomogeneities of extreme conductivity by boundary measurements: a theorem on continuous dependence. Arch. Rational Mech. Anal. 105 (1989) 299-326.

[27] D. Gilbarg and N.S. Trudinger, Elliptic partial differential equations of second order, Grundlehren der Mathematischen Wissenschaften 224. 2nd edition, Springer-Verlag, Berlin (1998).

[28] R. Griesmaier, An asymptotic factorization method for inverse electromagnetic scattering in layered media. SIAM J. Appl. Math. 68 (2008) 1378-1403.

[29] R. Griesmaier, Reciprocity gap music imaging for an inverse scattering problem in two-layered media. Inverse Probl. Imaging 3 (2009) 389-403.

[30] R. Griesmaier, Reconstruction of thin tubular inclusions in three-dimensional domains using electrical impedance tomography. SIAM J. Imaging Sci. 3 (2010) 340-362.

[31] R. Griesmaier and M. Hanke, An asymptotic factorization method for inverse electromagnetic scattering in layered media II: A numerical study. Contemp. Math. 494 (2008) 61-79.

[32] R. Griesmaier and M. Hanke, MUSIC-characterization of small scatterers for normal measurement data. Inverse Problems $\mathbf{2 5}$ (2009) 075012. 
[33] E. Iakovleva, S. Gdoura, D. Lesselier and G. Perrusson, Multistatic response matrix of a 3-D inclusion in half space and MUSIC imaging. IEEE Trans. Antennas Propag. 55 (2007) 2598-2609

[34] A.M. Il'in, Matching of asymptotic expansions of solutions of boundary value problems, Translations of Mathematical Monographs 102, translated by V. Minachin, American Mathematical Society, Providence, RI (1992).

[35] A. Kirsch and N. Grinberg, The Factorization Method for Inverse Problems, Oxford Lecture Ser. Math. Appl. 36. Oxford University Press, New York (2008).

[36] W. McLean, Strongly Elliptic Systems and Boundary Integral Equations. Cambridge University Press, Cambridge (2000).

[37] P. Monk, Finite Element Methods for Maxwell's Equations. Numer. Math. Sci. Comput. Oxford University Press, New York (2003).

[38] F. Murat and L. Tartar, H-convergence, Progress in Nonlinear Differential Equations and Their Applications 31, edited by A. Cherkaev and R. Kohn. Birkhäuser, Boston (1997).

[39] W.-K. Park and D. Lesselier, MUSIC-type imaging of a thin penetrable inclusion from its multi-static response matrix. Inverse Problems 25 (2009) 075002.

[40] W. Rudin, Real and complex analysis. McGraw-Hill Book Co., New York (1966).

[41] W. Rudin, Functional analysis. McGraw-Hill Series in Higher Mathematics. McGraw-Hill Book Co., New York (1973).

[42] D. Volkov, Numerical methods for locating small dielectric inhomogeneities. Wave Motion 38 (2003) $189-206$.

[43] C. Weber, Regularity theorems for Maxwell's equations. Math. Methods Appl. Sci. 3 (1981) 523-536. 